GSA Data Repository 2020083

\title{
Supplementary text for
}

Modeling the dynamics of a Late Triassic vertebrate extinction: the Adamanian/Revueltian faunal turnover, Petrified Forest National Park,

\section{$A Z$, USA}

Reilly F. Hayes ${ }^{1}$, Gavino Puggioni ${ }^{2}$, William G. Parker ${ }^{3}$, Catherine Tiley $^{1}$, Amanda Bednarick ${ }^{1}$, David E. Fastovsky ${ }^{1}$

${ }^{1}$ Department of Geosciences, University of Rhode Island, 9 E Alumni Avenue, Kingston, Rhode Island 02881, USA

${ }^{2}$ Department of Computer Science and Statistics, University of Rhode Island, 9 Greenhouse Road, Kingston, Rhode Island 02881, USA

${ }^{3}$ Division of Resource Management, Petrified Forest National Park, Box 2217, Petrified Forest, Arizona 86028, USA

\section{NOTES ON TAXON SAMPLING}

Diagnoses of fossils used in this analysis are current as of Summer 2018. As Parker and Martz (2011) observe, many taxa in the Chinle of Petrified Forest National Park (PEFO) persist through the Adamanian/Revueltian (A/R) faunal turnover: these include Crocodylomorpha, Coelophysoidea, Rauisuchidae, Shuvosauridae, Silesauridae, Sphenosuchia, Vancleavea campi, and Koskinonodon perfectus, following the recognition of Gee et al. (2017) and Gee and Parker 
(2017) that Apachesaurus gregorii likely represents a juvenile of this taxon. Because these taxa are not thought to originate or go extinct within the analytical time series, we exclude them from our analyses.

The Adamanian faunal assemblage is comprised by Acaenasuchus geoffreyi, Trilophosaurus sp., Calyptosuchus wellesi, Placerias hesternus, Desmatosuchus spurensis, Smilosuchus sp., Scutarx deltatylus, Poposaurus gracilis, Adamanasuchus eisenhardtae, Tecovasuchus chatterjeei, Acallosuchus rectori, and Crosbysaurus harrisae, while the Revueltian assemblage is comprised by Machaeroprosopus sp., Chindesaurus bryansmalli, and Revueltosaurus callenderi, and Rioarribasuchus chamaensis. Additionally, while not confined to the Adamanian or Revueltian, the pattern of fossil occurrences of Typothorax sp. and Paratypothorax sp. (Parker and Martz, 2011) suggests that these taxa originated sometime during the analytical time series. Each of the taxa above must meet three criteria to be included in this analysis:

1. Fossils must come from those Chinle localities where published $\mathrm{U}-\mathrm{Pb}$ dates accommodate rigorous age estimation in a Bchron age-depth model (i.e. Petrified Forest National Park, the Placerias Quarry (Camp and Welles, 1956), and the Hayden Quarry (Irmis et al., 2007)). Age constraints based on fossils or lithology, often established at those Chinle localities where geochronologic dates are unavailable, do not rise to the level of temporal precision permissible for this analysis.

2. The Alroy (2014) algorithm requires that each taxon have at least three fossil occurrences of different ages: two to define a temporal range, and at least one between them to define a frequency within that range. We therefore exclude all taxa known from fewer than three total fossil occurrences at PEFO, the Placerias Quarry, and the Hayden 
Quarry: these include Tecovasuchus chatterjeei (PFV 211), Acallosuchus rectori (PFV 124), and Crosbysaurus harrisae (PFV 122). We also exclude on these grounds Rioarribasuchus chamaensis (PFV 075, PFV 366, and the Hayden Quarry) because our Bchron age-depth model frequently reconstructs two or more of these localities as contemporaneous, pushing the taxon below the analytical threshold.

3. Fossils must occur in localities correlable to U-Pb dated beds via continuously-exposed outcrop. We include seventy-one Petrified Forest fossil localities in this analysis, but exclude fifteen additional localities that do not meet this criterion. General stratigraphic positions can be established for these additional localities per the stratigraphy of Martz and Parker (2010; see also Parker and Martz, 2011); however, the uncertainty associated with those correlations-information required to integrate a locality into our age-depth model-cannot be tallied into a non-arbitrary cumulative term, as can those associated with correlations constructed along continuous outcrop. Accordingly, we exclude from the analysis Scutarx deltatylus (occurrences at PFV 224, PFV 169, PFV 304, and PFV 355, but the latter three of these cannot be correlated to dated beds of Ramezani et al. (2011) with sufficient stratigraphic precision) and Poposaurus gracilis (occurrences at the Placerias Quarry, PFV 161, and PFV 336, but the last of these cannot be correlated with sufficient precision).

We therefore attribute to the Adamanian extinction event the extinctions of Acaenasuchus geoffreyi, Trilophosaurus sp., Calyptosuchus wellesi, Placerias hesternus, Desmatosuchus spurensis, and Smilosuchus sp., and to the Revueltian origination event the originations of Machaeroprosopus sp., Chindesaurus bryansmalli, and Revueltosaurus 
callenderi. We exclude Typothorax sp. and Paratypothorax sp. from this analytical definition of the Revueltian origination because Adamanian-aged fossils belonging to these taxa exist.

Many taxa included in our analysis are not endemic to the study area (northeastern Arizona and north-central New Mexico), yet those fossil occurrences contained within our data set (at PEFO, the Placerias Quarry, and the Hayden Quarry) represent the only geochronologically-controlled portion of their record. It is possible that younger fossils may exist elsewhere, but this cannot be demonstrated with current evidence.

The $A / R$ turnover represents a transition between assemblages of genera, not species, and moreover, many fossils in PEFO are not diagnosable to the specific level; as is common with fossil vertebrate taxa, we therefore perform our analyses at the generic level. Additionally, because the Alroy (2014) and Solow (2016) methods require only the knowledge that a genus was present at a given time, our analyses do not consider abundance, nor do we attempt to discriminate between fossils belonging to unique individuals at a given locality. All locality data are permanently stored at Petrified Forest National Park. Table S7 lists voucher numbers for all fossils included in the analysis. 


\section{AGE-DEPTH MODELING IN BCHRON}

The methods of Alroy (2014) and Solow (2016) require a record of known fossil ages to reconstruct extinction probability. Notably, however, these methods provide no means of accounting for the temporal uncertainty inherent in such records in deep time. It was therefore necessary to establish a mechanism for assessing the precision with which absolute ages could be interpolated for fossils throughout the thickness of the dated Chinle sequence exposed in PEFO.

For each PEFO fossil locality, we calculated a distribution of plausible ages using a pair of age-depth models constructed in the R package Bchron (v. 4.3.0, Haslett and Parnell, 2008). We defined separate models for northern (Figure S1) and southern (Figure S2) PEFO for practicality, as stratigraphic correlations can be most precisely defined between $\mathrm{U}-\mathrm{Pb}$ dates and those fossils situated closest geographically. Ages of fossils in northern PEFO could thus be best determined with a model employing the date KWI (Devil's Playground, northern PEFO;

Ramezani et al., 2011), and southern fossils with a model employing P-57C (Mountain Lion Mesa, southern PEFO; Nordt et al., 2015). In cases when we could not determine exactly which bed at a locality yielded fossils, we reported that stratigraphic uncertainty to the age-depth model, which accordingly produced a broader distribution of possible ages for all fossils at that locality. In those cases when we could assign particular fossils at a locality to distinct beds, we entered those beds, with their corresponding stratigraphic uncertainties, separately in the model. 
Inputs for the Bchronology function, used to build northern and southern PEFO age-depth models, are respectively available in Tables S1 and S2. Age inputs ("ages" and "ageSds" arguments) are scaled down by $10^{3}$, but Bchronology scales them to their true magnitude as the ageScaleVal argument defaults to 1000. Stratigraphic inputs ("position" and "thickness") are derived from the original field notes supporting the correlations of Ramezani et al. (2011), in addition to the positions Atchley et al. (2014) and Nordt et al. (2015) report for the dates SS-7 and $\mathrm{P} 57-\mathrm{C}$. Because all ages are derived from a U-Pb isotopic system, we set the calibration curves ("calCurves") argument to "normal," following the instruction of Bchron documentation for non-14C ages. Each age model was run for 1,000,000 iterations, with a burn-in period of 200,000 iterations and one iteration kept every 800 steps beyond the burn-in. Diagnostics (convergence checks and posterior outlier probability by date) of the age models are available in Tables S3 and S4.

We estimated ages for the Placerias and Hayden Quarries by respectively sampling ages in Bchron from the dates AB0513-2 (Ramezani et al., 2014) and Hayden 2 (Irmis et al., 2011). This approach, by contrast to an age-depth model, is justified because these dates were sampled directly from fossiliferous beds at both localities. It should be noted, however, that additional fossiliferous horizons exist at the Hayden Quarry beyond that containing Hayden 2. We thus assumed that the broad analytical uncertainty associated with that date $( \pm 0.7 \mathrm{Ma})$ encompasses the complete depositional age of the Hayden Quarry.

We estimated ages for these localities first by passing the inputs given in Tables S5 and S6 to the function BchronCalibrate to calibrate the dates. We then passed the subsequent outputs to the function sampleAges to generate ages. BchronCalibrate was run with all arguments set to 
their default values, and sampleAges with the "n_sample" argument set to provide 1000 age estimates. 


\section{NOTES ON MODEL INTERPRETATION}

The model employed in this study differs somewhat from that of Alroy (2014) and Solow (2016) in the nature of its fossil data. The simulated data presented in Alroy (2014) have a temporal precision unavailable here, as there is no uncertainty of which time bin ought to contain a given taxon occurrence. The same problem is present in the alternative approach of Solow (2016). We address the issue through our age-depth model. The model builds age distributions reflecting the temporal uncertainty around each fossil, and our analyses account fairly for that uncertainty by considering the full breadth of these distributions in constructing posterior probability densities of extinction/origination (by averaging results from 1000 runs of the Alroy (2014) and Solow (2016) algorithms, with each run corresponding to a unique combination of draws of fossil ages from the distributions produced by the age-depth models).

Figure 2 (main text) illustrates the density of the probability that taxa go extinct or originate through time. Extinction or origination is most likely where the pair of curves (top and bottom respectively representing analyses run with the Alroy (2014) and Solow (2016) methods) for each taxon are highest, and less so where they are lower. The reason that most curves extend far throughout the time series is the sparseness of fossil data. For example, because Placerias has few fossil occurrences, the algorithms conservatively predict a broader distribution of possible extinction times. This gives the long flat curve pictured in Figure 2 . What it does not signify is that Placerias must have survived for an anomalously long time after the last fossil; all it indicates is that the model cannot constrain the time of that extinction with a great degree of certainty. If we consider the curves of all taxa, rather than Placerias alone, 
much overlap is evident. Clearly, there is some probability that extinctions and/or originations were synchronous.

We carry the analysis further by quantifying the exact probability of synchroneity. The probability that extinctions and/or originations were synchronous is simply the joint probability that those events happened in a single analytical interval. This joint probability can be straightforwardly calculated as the product of the respective areas under the densities of those taxa, as pictured in Figure 2. The summation of these joint probabilities across the full time series gives the total probability that any number of extinctions and/or originations were synchronous at any time in the analytical window, regardless of when exactly that time was. Table 1 and Figure 3 (main text) report these overall probabilities. 


\section{TESTING OF THE ASSUMPTION OF UNIFORM FOSSIL RECOVERY}

The Alroy (2014) and Solow (2016) methods assume that fossil sampling probability remains uniform through the stratigraphic record of each taxon-a condition far from certain in the terrestrial fluvial system represented by the Chinle. We test this assumption via the uniform probability plot correlation coefficient (PPCC) approach of Vogel et al. (2009; see also Wang et al., 2009). This test assesses how well a stratigraphic pattern of fossils fits a uniform distribution. Outputs of tests such as this are typically interpreted graphically, but because of the prohibitively large number of iterations we performed (11,000; one test per interpolated Chinle chronology per taxon), we instead automated the hypothesis testing procedure described by Vogel and Kroll (1989, p. 343) to assess the realism of assuming uniformity.

Among the 1000 stochastically interpolated Chinle chronologies provided by our age-depth model, the test cannot consistently distinguish between uniform and fossil patterns of Acaenasuchus (100.0\% of chronologies accord with uniform distribution), Calyptosuchus (99.9\%), Desmatosuchus (93.3\%), Machaeroprosopus (99.9\%), Paratypothorax (100.0\%), Placerias (100.0\%), Smilosuchus (100.0\%), and Trilophosaurus (100.0\%). Notably, this list encompasses the full Adamanian assemblage, as well as Machaeroprosopus, the diagnostic taxon of the Revueltian biozone.

Uniformity can be rejected for the three remaining taxa (Chindesaurus, $18.0 \%$ of chronologies accord with uniform distribution; Revueltosaurus, 4.10\%; Typothorax, 0.70\%) because their fossil occurrences skew young. Because we do not attribute Typothorax to the Revueltian biozone, only the former two taxa bear upon the results of the analysis. If we therefore ignore these taxa while calculating the probability that Adamanian extinctions 
coincided with Revueltian originations (here, that of Machaeroprosopus alone), the result is $3.68 \times 10^{-12}$. Because this figure compels us to reject the null hypothesis-just as we would without ignoring Chindesaurus and Revueltosaurus - we may conclude that violations of assumed uniform fossil recovery in this study system are not so egregious as to meaningfully distort our analysis. 


\section{ADDITIONAL ANALYSIS ASSUMING UNDERSAMPLING OF TAXA}

Alroy (2014) recognized that if a taxon existed prior to the beginning of the stratigraphic record at hand-a circumstance he dubs "undersampling" of that taxon-its prior extinction probability $P(E)$ would be too large. Given our uncertainty of whether this dissonance between the observed and true ranges ever truly exists, Alroy (2014) suggested doubling the range term $R$ of the prior as a fair correction to the problem. We took this advice, and performed a more conservative iteration our Alroy-based analysis with the prior:

$$
P(E)=-\log (0.5) / 2 R
$$

This is not an issue for the Solow (2016) method, which employs a prior determined independently of range data.

Results of this additional analysis accord with those of the previous Solow- and Alroy-based analyses: the probabilities of a singular Adamanian extinction event, singular Revueltian origination event, and synchronous A/R turnover are infinitesimal across all three (Tables S8S11; Figures S3, S4). 


\section{REFERENCES CITED}

Atchley S.C., Nordt L.C., Dworkin S.I., Ramezani J., Parker W.G., Ash S.R., and Bowring S.A., 2014, A linkage among Pangean tectonism, cyclic alluviation, climate change, and biologic turnover in the Late Triassic: The record from the Chinle Formation, southwestern United States: Journal of Sedimentary Research, v. 83, no. 12, p. 1147-1161, https://doi.org/10.2110/jsr.2013.89.

Alroy, J., 2014, A simple Bayesian method of inferring extinction: Paleobiology v. 40, no. 4, p. 584-607, https://doi.org:10.1666/13074.

Alroy, J., 2016, A simple Bayesian method of inferring extinction: Reply: Ecology, v. 97, no. 3, p. 798-800, https://doi.org/10.1890/15-1711.

Camp, C., and Welles, S.P., 1956, Triassic Dicynodont Reptiles: Memoirs of the University of California, University of California Press, Berkeley, CA, v. 13, p. 255 - 348.

Haslett, J., and Parnell, A.C., 2008, A simple monotone process with application to radiocarbondated depth chronologies: Journal of the Royal Statistical Society: Series C (Applied Statistics), v. 57, no. 4, p. 399-418, https://doi.org/10.1111/j.1467-9876.2008.00623.x.

Gee, B.M. and Parker, W.G., 2017, A juvenile Koskinonodon perfectus (Temnospondyli, Metoposauridae) from the Upper Triassic of Arizona and its implications for the taxonomy of North American metoposaurids: Journal of Paleontology, v. 91, no. 5, p.1047-1059, https://doi.org/10.1017/jpa.2017.18. 
Gee, B.M., Parker, W.G., and Marsh, A.D., 2017, Microanatomy and paleohistology of the intercentra of North American metoposaurids from the Upper Triassic of Petrified Forest National Park (Arizona, USA) with implications for the taxonomy and ontogeny of the group: PeerJ, v. 5, p.e3183, https://doi.org/10.7717/peerj.3183.

Irmis, R.B., Nesbitt, S.J., Padian, K., Smith, N.D., Turner, A.H., Woody, D., and Downs, A., 2007, A Late Triassic dinosaurimorph assemblage from New Mexico and the rise of dinosaurs: Science, v. 317, p. 358-361, doi:10.1126/science.1143325.

Irmis, R.B., Mundil, R., Martz, J.W., and Parker, W.G., 2011, High-resolution U-Pb ages from the Upper Triassic Chinle Formation (New Mexico, USA) support a diachronous rise of dinosaurs: Earth and Planetary Science Letters, v. 309, p. 258-267, no. 3-4, https://doi.org/10.1016/j.epsl.2011.07.015.

Nordt, L., Atchely, S., and Dworkin, S., 2015, Collapse of the late Triassic megamonsoon in western equatorial Pangea, present-day American Southwest: Geological Society of America Bulletin, v. 127, no. 11-12, p. 1798 - 1815, https://doi.org/10.1130/b31186.1.

Parker, W.G., and Martz, J.W., 2011, The Late Triassic (Norian) Adamanian-Revueltian tetrapod faunal transition in the Chinle Formation of Petrified Forest National Park, Arizona: Earth and Environmental Science Transactions of the Royal Society of Edinburgh, v. 101, no. 3-4, p. 231-260, https://doi.org/10.1017/s1755691011020020.

Ramezani, J., Fastovsky, D.E., and Bowring, S.A., 2014, Revised chronostratigraphy of the lower Chinle Formation strata in Arizona and New Mexico (USA): High-precision U-Pb 
geochronological constraints on the Late Triassic evolution of dinosaurs: American Journal of Science, v. 314, p. 981-1008, https://doi.org/10.2475/06.2014.01.

Solow, A.R., 2016, A simple Bayesian method of inferring extinction: Comment: Ecology, v. 97, no. 3, p. 796-798, https://doi.org/10.1890/15-1711.

Vogel, R. M., and Kroll, C. N., 1989, Low-flow frequency analysis using probability-plot correlation coefficients: Journal of water resources planning and management, v. 115, no. 3, p. 338-357, https://doi.org/10.1061/(asce)0733-9496(1989)115:3(338).

Vogel, R. M., J. R. M. Hosking, C. S. Elphick, D. L. Roberts, and Reed, J.M., 2009, Goodness of fit of probability distributions for sightings as species approach extinction: Bulletin of Mathematical Biology, v. 71, p. 701-719, https://doi.org/10.1007/s11538-008-9377-3.

Wang, S.C., Chudzicki, D.J., and Everson, P.J., 2009, Optimal estimators of the position of a mass extinction when recovery potential is uniform: Paleobiology, v. 35, no. 3, p. 447-459, https://doi.org/10.1666/0094-8373-35.3.447. 
TABLE S1. GEOCHRONOLOGIC DATA USED FOR BCHRON AGE-DEPTH MODEL OF NORTHERN PETRIFIED FOREST NATIONAL PARK.

\begin{tabular}{llllll}
\hline \hline id & ages & ageSds & position & thickness & calCurves \\
\hline BFB & 209926 & 72 & 0 & 1.25 & normal \\
GPU & 213124 & 69 & 101.01 & 0.75 & normal \\
KWI & 213870 & 78 & 109.545 & 1.28 & normal \\
GPL & 218017 & 88 & 140.045 & 1.92 & normal \\
SBJ & 219317 & 80 & 154.32 & 0.37 & normal \\
SS-7 & 220123 & 68 & 185.075 & 0.5 & normal \\
TPS & 223036 & 59 & 189.125 & 0.76 & normal \\
SS-28 & 225185 & 79 & 241.075 & 0.5 & normal
\end{tabular}


TABLE S2. GEOCHRONOLOGIC DATA USED FOR BCHRON AGE-DEPTH MODEL OF SOUTHERN PETRIFIED FOREST NATIONAL PARK.

\begin{tabular}{llllll}
\hline \hline id & ages & ageSds & position & thickness & calCurves \\
\hline BFB & 209926 & 72 & 0 & 1.25 & normal \\
GPU & 213124 & 69 & 101.01 & 0.75 & normal \\
P57-C & 213630 & 130 & 109.575 & 0.5 & normal \\
GPL & 218017 & 88 & 140.045 & 1.92 & normal \\
SBJ & 219317 & 80 & 154.32 & 0.37 & normal \\
SS-7 & 220123 & 68 & 185.075 & 0.5 & normal \\
TPS & 223036 & 59 & 189.125 & 0.76 & normal \\
SS-28 & 225185 & 79 & 241.075 & 0.5 & normal
\end{tabular}


TABLE S3. DIAGNOSTICS OF NORTHERN PEFO AGE-DEPTH MODEL.

\begin{tabular}{|c|c|c|c|}
\hline \multicolumn{2}{|c|}{ Convergence check } & \multicolumn{2}{|c|}{ Posterior outlier probability by date } \\
\hline Item & p-value & Date & Probability \\
\hline SS-28 & 0.01079 & BFB & 0.011 \\
\hline $\mathrm{BFB}$ & 0.04959 & & \\
\hline Outlier 1 & 0.05388 & GPU & 0.007 \\
\hline KWI & 0.05895 & & \\
\hline GPU & 0.06749 & KWI & 0.009 \\
\hline SBJ & 0.10622 & & \\
\hline RateVar & 0.10981 & GPL & 0.017 \\
\hline Outlier 5 & 0.16353 & & \\
\hline GPL & 0.26781 & SBJ & 0.014 \\
\hline Outlier 4 & 0.27108 & & \\
\hline TPS & 0.31382 & SS-7 & 0.012 \\
\hline Outlier 7 & 0.33808 & & \\
\hline Outlier 2 & 0.35450 & TPS & 0.008 \\
\hline Outlier 8 & 0.35450 & & \\
\hline Outlier 6 & 0.35820 & SS-28 & 0.007 \\
\hline RateMean & 0.41734 & & \\
\hline Outlier 3 & 0.42872 & & \\
\hline SS-7 & 0.43179 & & \\
\hline
\end{tabular}


TABLE S4. DIAGNOSTICS OF SOUTHERN PEFO AGE-DEPTH MODEL.

\begin{tabular}{lccc}
\hline \hline & Convergence check & \multicolumn{2}{c}{ Posterior outlier probability by date } \\
\cline { 2 - 4 } \multicolumn{1}{c}{ Item } & p-value & Date & Probability \\
\hline Outlier 7 & 0.01391 & & 0.010 \\
TPS & 0.01578 & GPU & 0.011 \\
Outlier 2 & 0.02498 & & \\
GPL & 0.03077 & P57-C & 0.008 \\
SS-7 & 0.04116 & & \\
Outlier 3 & 0.04132 & GPL & 0.010 \\
P57-C & 0.10662 & & \\
SS-28 & 0.21414 & SBJ & 0.009 \\
RateMean & 0.29593 & & \\
Outlier 6 & 0.29694 & SS-7 & 0.012 \\
RateVar & 0.30071 & & 0.011 \\
Outlier 4 & 0.30129 & TPS & \\
Outlier 8 & 0.35820 & & 0.014 \\
GPU & 0.37987 & SS-28 & \\
BFB & 0.39042 & & \\
SBJ & 0.39612 & & \\
Outlier 1 & 0.42872 & & \\
Outlier 5 & 0.49709 & &
\end{tabular}


TABLE S5. GEOCHRONOLOGIC DATA USED FOR MODELING AGE OF PLACERIAS QUARRY IN BCHRON.

\begin{tabular}{lcccc}
\hline \hline id & ages & ageSds & position & calCurves \\
\hline AB0513-2 & 219390 & 120 & 0 & normal
\end{tabular}


TABLE S6. GEOCHRONOLOGIC DATA USED FOR MODELING AGE OF HAYDEN QUARRY IN BCHRON.

\begin{tabular}{lcccc}
\hline \hline id & ages & ageSds & position & calCurves \\
\hline Hayden 2 & 211900 & 700 & 0 & normal
\end{tabular}


TABLE S7. VOUCHER SPECIMENS AND ASSOCIATED FOSSIL LOCALITIES.

\begin{tabular}{|c|c|c|c|c|}
\hline Taxon & Locality & Bchron model & $\begin{array}{l}\text { Position in age- } \\
\text { depth model (m) }\end{array}$ & Voucher \# \\
\hline \multirow{4}{*}{$\begin{array}{l}\text { Acaenasuchus geoffreyi } \\
\text { (Aetosauria) }\end{array}$} & PFV122 & Southern PEFO & $190.44 \pm 1.35$ & PEFO 20358 \\
\hline & PFV211 & Southern PEFO & $175.55+2.54$ & PEFO 16621 \\
\hline & Placerias & Placerias & 0.00 & MNA V36668 \\
\hline & Quarry & Quarry & & \\
\hline \multirow{8}{*}{$\begin{array}{l}\text { Calyptosuchus wellesi } \\
\text { (Aetosauria) }\end{array}$} & PFV111 & Southern PEFO & $203.13 \pm 1.67$ & UCMP 126856 \\
\hline & PFV112 & Southern PEFO & $204.66 \pm 2.02$ & UCMP 126854 \\
\hline & PFV161 & Southern PEFO & $170.98 \pm 3.57$ & UCMP 139492 \\
\hline & PFV162 & Southern PEFO & $169.61 \pm 1.81$ & UCMP 126844 \\
\hline & PFV165 & Southern PEFO & $164.51 \pm 2.81$ & UCMP 126943 \\
\hline & PFV167 & Southern PEFO & $146.76 \pm 3.3$ & UCMP 126882 \\
\hline & PFV396 & Southern PEFO & $204.39+4.38$ & PEFO 38265 \\
\hline & PFV445 & Southern PEFO & $201.52 \pm 4.33$ & PEFO 38612 \\
\hline \multirow{10}{*}{$\begin{array}{l}\text { Desmatosuchus } \\
\text { spurensis } \\
\text { (Aetosauria) }\end{array}$} & PFV113 & Southern PEFO & $185.945 \pm 2.92$ & PEFO 5038 \\
\hline & PFV167 & Southern PEFO & $146.755 \pm 3.30$ & UCMP 126885 \\
\hline & PFV178 & Southern PEFO & $127.275 \pm 3.51$ & UCMP 126976 \\
\hline & PFV198 & Southern PEFO & $202.47 \pm 5.56$ & PEFO 31177 \\
\hline & PFV202 & Southern PEFO & $190.47 \pm 1.83$ & PEFO 23338 \\
\hline & PFV211 & Southern PEFO & $175.55 \pm 2.54$ & PEFO 38402 \\
\hline & PFV212 & Southern PEFO & $173.90 \pm 1.62$ & PEFO 26668 \\
\hline & PFV267 & Southern PEFO & $161.03 \pm 0.47$ & PEFO 34935 \\
\hline & Placerias & Placerias & 0.00 & UCMP 78748 \\
\hline & Quarry & Quarry & & \\
\hline \multirow[t]{6}{*}{$\begin{array}{l}\text { Paratypothorax } \\
\text { (Aetosauria) }\end{array}$} & PFV037 & $\begin{array}{l}\text { Northern } \\
\text { PEFO }\end{array}$ & $96.60 \pm 1.32$ & UCMP 139486 \\
\hline & PFV071 & Southern PEFO & $71.19 \pm 0.35$ & UCMP 139958 \\
\hline & PFV097 & Northern & $137.73 \pm 1.32$ & UCMP 129995 \\
\hline & PFV167 & Southern PEFO & $146.755 \pm 3.30$ & PEFO 35003 \\
\hline & PFV272 & Southern PEFO & $117.34 \pm 1.82$ & PEFO 31206 \\
\hline & PFV366 & Southern PEFO & $101.76 \pm 2.81$ & PEFO 35263 \\
\hline \multirow[t]{3}{*}{$\begin{array}{l}\text { Typothorax coccinarum } \\
\text { (Aetosauria) }\end{array}$} & PFV037 & $\begin{array}{l}\text { Northern } \\
\text { PEFO }\end{array}$ & $96.60 \pm 1.32$ & PEFO 5039 \\
\hline & PFV040 & $\begin{array}{l}\text { Northern } \\
\text { PEFO }\end{array}$ & $19.91 \pm 3.28$ & PEFO 36757 \\
\hline & PFV060 & Southern PEFO & $91.83+1.42$ & PEFO 34882 \\
\hline
\end{tabular}




\begin{tabular}{|c|c|c|c|c|}
\hline Taxon & Locality & Bchron model & $\begin{array}{l}\text { Position in age- } \\
\text { depth model }(\mathrm{m})\end{array}$ & Voucher \# \\
\hline \multirow{22}{*}{$\begin{array}{l}\text { Typothorax coccinarum } \\
\text { (Aetosauria) }\end{array}$} & PFV070 & Southern PEFO & $71.19 \pm 0.35$ & PEFO 23388 \\
\hline & PFV071 & Southern PEFO & $71.19 \pm 0.35$ & PEFO 34851 \\
\hline & PFV075 & Southern PEFO & $92.12 \pm 2.14$ & PEFO 36779 \\
\hline & PFV089 & Southern PEFO & $112.69 \pm 1.28$ & PEFO 34869 \\
\hline & PFV092 & Southern PEFO & $102.98 \pm 3.99$ & PEFO 34214 \\
\hline & PFV094 & Southern PEFO & $105.35 \pm 2.76$ & UCMP 126855 \\
\hline & PFV097 & Northern & $137.73 \pm 1.32$ & PEFO 34918 \\
\hline & & PEFO & & \\
\hline & PFV121 & Southern PEFO & $173.87 \pm 3.29$ & PEFO 34213 \\
\hline & PFV215 & Northern & $15.30 \pm 2.97$ & PEFO 16668 \\
\hline & & PEFO & & \\
\hline & PFV227 & Southern PEFO & $113.06 \pm 1.06$ & PEFO 35018 \\
\hline & PFV231 & Northern & $25.00 \pm 2.30$ & PEFO 33980 \\
\hline & & PEFO & & \\
\hline & PFV268 & Southern PEFO & $117.845 \pm 1.15$ & PEFO 26702 \\
\hline & PFV290 & Southern PEFO & $120.54 \pm 2.35$ & PEFO 34884 \\
\hline & PFV295 & Southern PEFO & $107.77 \pm 1.58$ & PEFO 34280 \\
\hline & PFV326 & $\begin{array}{l}\text { Northern } \\
\text { PEFO }\end{array}$ & $34.75 \pm 1.49$ & PEFO 38654 \\
\hline & PFV349 & Southern PEFO & $93.24 \pm 1.31$ & PEFO 34847 \\
\hline & PFV367 & $\begin{array}{l}\text { Northern } \\
\text { PEFO }\end{array}$ & $129.49 \pm 0.97$ & PEFO 34918 \\
\hline & PFV371 & $\begin{array}{l}\text { Northern } \\
\text { PEFO }\end{array}$ & $97.75 \pm 2.38$ & PEFO 35131 \\
\hline & $\begin{array}{l}\text { Hayden } \\
\text { Quarry }\end{array}$ & Hayden Quarry & 0.00 & GR 229 \\
\hline \multirow[t]{6}{*}{$\begin{array}{l}\text { Machaeroprosopus } \\
\text { (Phytosauria) }\end{array}$} & PFV037 & $\begin{array}{l}\text { Northern } \\
\text { PEFO }\end{array}$ & $96.60 \pm 1.32$ & PEFO 5034 \\
\hline & PFV040 & $\begin{array}{l}\text { Northern } \\
\text { PEFO }\end{array}$ & $19.91 \pm 3.28$ & UCMP 126726 \\
\hline & PFV042 & $\begin{array}{l}\text { Northern } \\
\text { PEFO }\end{array}$ & $28.79 \pm 2.37$ & PEFO 31219 \\
\hline & PFV075 & Southern PEFO & $92.12 \pm 2.14$ & UCMP 126993 \\
\hline & PFV271 & Southern PEFO & $94.29 \pm 1.81$ & PEFO 31205 \\
\hline & PFV295 & Southern PEFO & $107.77 \pm 1.58$ & PEFO 31207 \\
\hline \multirow[t]{3}{*}{$\begin{array}{l}\text { Smilosuchus } \\
\text { (Phytosauria) }\end{array}$} & PFV097 & $\begin{array}{l}\text { Northern } \\
\text { PEFO }\end{array}$ & $137.73 \pm 1.32$ & UCMP 26688 \\
\hline & PFV098 & $\begin{array}{l}\text { Northern } \\
\text { PEFO }\end{array}$ & $133.43 \pm 1.19$ & UCMP 27181a \\
\hline & PFV113 & Southern PEFO & $185.95 \pm 2.92$ & UCMP 139554 \\
\hline
\end{tabular}




\begin{tabular}{|c|c|c|c|c|}
\hline Taxon & Locality & Bchron model & $\begin{array}{l}\text { Position in age- } \\
\text { depth model (m) }\end{array}$ & Voucher \# \\
\hline \multirow{8}{*}{$\begin{array}{l}\text { Smilosuchus } \\
\text { (Phytosauria) }\end{array}$} & PFV122 & Southern PEFO & $190.44 \pm 1.34$ & PEFO 5083 \\
\hline & PFV150 & Southern PEFO & $165.23 \pm 2.29$ & PEFO 34869 \\
\hline & PFV142 & Southern PEFO & $201.61 \pm 5.40$ & PEFO 31156 \\
\hline & PFV161 & Southern PEFO & $170.98 \pm 3.57$ & PEFO 34921 \\
\hline & PFV177 & Southern PEFO & $129.49 \pm 2.54$ & UCMP 129809 \\
\hline & PFV178 & Southern PEFO & $127.28 \pm 3.51$ & PEFO 34866 \\
\hline & PFV182 & Southern PEFO & $201.055 \pm 1.38$ & PEFOF 26682 \\
\hline & PFV268 & Southern PEFO & $117.845 \pm 1.15$ & PEFO 31203 \\
\hline \multirow[t]{6}{*}{$\begin{array}{l}\text { Chindesaurus } \\
\text { bryansmalli (Dinosauria) }\end{array}$} & PFV018 & $\begin{array}{l}\text { Northern } \\
\text { PEFO }\end{array}$ & $21.12 \pm 1.04$ & PEFO 4849 \\
\hline & PFV020 & $\begin{array}{l}\text { Northern } \\
\text { PEFO }\end{array}$ & $29.15 \pm 0.51$ & PEFO 10395 \\
\hline & PFV089 & Southern PEFO & $112.69 \pm 1.28$ & PEFO 34875 \\
\hline & PFV231 & $\begin{array}{l}\text { Northern } \\
\text { PEFO }\end{array}$ & $25.00 \pm 2.30$ & PEFO 33982 \\
\hline & PFV332 & $\begin{array}{l}\text { Northern } \\
\text { PEFO }\end{array}$ & $23.67 \pm 7.62$ & PEFO 34583 \\
\hline & $\begin{array}{l}\text { Hayden } \\
\text { Quarry }\end{array}$ & Hayden Quarry & 0.00 & GR 226 \\
\hline \multirow[t]{5}{*}{$\begin{array}{l}\text { Revueltosaurus } \\
\text { callenderi (Archosauria) }\end{array}$} & PFV040 & $\begin{array}{l}\text { Northern } \\
\text { PEFO }\end{array}$ & $19.91 \pm 3.28$ & PEFO 34169 \\
\hline & PFV089 & Southern PEFO & $112.69 \pm 1.28$ & PEFO 36759 \\
\hline & PFV215 & $\begin{array}{l}\text { Northern } \\
\text { PEFO }\end{array}$ & $15.30 \pm 2.97$ & PEFO 16671 \\
\hline & PFV231 & $\begin{array}{l}\text { Northern } \\
\text { PEFO }\end{array}$ & $25.00 \pm 2.30$ & PEFOF 33991 \\
\hline & PFV297 & $\begin{array}{l}\text { Northern } \\
\text { PEFO }\end{array}$ & $22.98 \pm 0.38$ & PEFO 33787 \\
\hline \multirow{5}{*}{$\begin{array}{l}\text { Trilophosaurus } \\
\text { (Archosauria) }\end{array}$} & PFV122 & Southern PEFO & $190.44 \pm 1.35$ & PEFO 3893 \\
\hline & PFV191 & Southern PEFO & $151.58 \pm 1.21$ & PEFO 31165 \\
\hline & PFV396 & Southern PEFO & $204.39 \pm 4.38$ & PEFO 38355 \\
\hline & Placerias & Placerias & 0.00 & UCMP 32390 \\
\hline & Quarry & Quarry & & \\
\hline \multirow[t]{4}{*}{$\begin{array}{l}\text { Placerias hesternus } \\
\text { (Dicynodonta) }\end{array}$} & PFV098 & $\begin{array}{l}\text { Northern } \\
\text { PEFO }\end{array}$ & $133.43 \pm 1.19$ & UCMP 26682 \\
\hline & PFV113 & Southern PEFO & $185.945 \pm 2.92$ & UCMP 139463 \\
\hline & PFV124 & Southern PEFO & $188.795 \pm 2.29$ & UCMP 27095 \\
\hline & $\begin{array}{l}\text { Placerias } \\
\text { Quarry }\end{array}$ & $\begin{array}{l}\text { Placerias } \\
\text { Quarry }\end{array}$ & 0.00 & MNA PI 2770 \\
\hline
\end{tabular}


TABLE S8. POSTERIOR PROBABILITY OF SYNCHRONOUS ADAMANIAN EXTINCTION EVENT, REVUELTIAN ORIGINATION

EVENT, AND A/R TURNOVER CALCULATED WITH ALROY (2014) METHOD ASSUMING UNDERSAMPLING OF ALL TAXA

\begin{tabular}{lc}
\hline \multicolumn{1}{c}{ Event } & Posterior Probability \\
\hline Adamanian extinction & $2.02 \times 10^{-10}$ \\
Revueltian origination & $2.93 \times 10^{-4}$ \\
A/R turnover & $5.91 \times 10^{-14}$
\end{tabular}


TABLE S9. AVERAGE POSTERIOR EXTINCTION PROBABILITIES GENERATED FOR ACAENASUCHUS, TRILOPHOSAURUS, CALYPOTSUCHUS, AND PLACERIAS BY ALROY (2014) AND SOLOW (2016) METHODS. "NO UNDERSAMPLING" AND "UNDERSAMPLING" DENTOED RESPECTIVELY BY "NU" AND "U".

\begin{tabular}{|c|c|c|c|c|c|c|c|c|c|c|c|c|}
\hline \multirow{2}{*}{$\begin{array}{c}\text { Interval } \\
\text { (Ma) }\end{array}$} & \multicolumn{3}{|c|}{ Acaenasuchus } & \multicolumn{3}{|c|}{ Trilophosaurus } & \multicolumn{3}{|c|}{ Calyptosuchus } & \multicolumn{3}{|c|}{ Placerias } \\
\hline & Solow & $\begin{array}{l}\text { Alroy } \\
\text { (NU) }\end{array}$ & $\begin{array}{l}\text { Alroy } \\
\text { (U) }\end{array}$ & Solow & $\begin{array}{l}\text { Alroy } \\
\text { (NU) }\end{array}$ & $\begin{array}{l}\text { Alroy } \\
\text { (U) }\end{array}$ & Solow & $\begin{array}{l}\text { Alroy } \\
\text { (NU) }\end{array}$ & $\begin{array}{l}\text { Alroy } \\
\text { (U) }\end{array}$ & Solow & $\begin{array}{l}\text { Alroy } \\
\text { (NU) }\end{array}$ & $\begin{array}{l}\text { Alroy } \\
\text { (U) }\end{array}$ \\
\hline 225.185 & 0 & 0 & 0 & 0 & 0 & 0 & 0 & 0 & 0 & 0 & 0 & 0 \\
\hline 225.085 & 0 & 0 & 0 & 0 & 0 & 0 & 0 & 0 & 0 & 0 & 0 & 0 \\
\hline 224.985 & 0 & 0 & 0 & 0 & 0 & 0 & 0 & 0 & 0 & 0 & 0 & 0 \\
\hline 224.885 & 0 & 0 & 0 & 0 & 0 & 0 & 0 & 0 & 0 & 0 & 0 & 0 \\
\hline 224.785 & 0 & 0 & 0 & 0 & 0 & 0 & 0 & 0 & 0 & 0 & 0 & 0 \\
\hline 224.685 & 0 & 0 & 0 & 0 & 0 & 0 & 0 & 0 & 0 & 0 & 0 & 0 \\
\hline 224.585 & 0 & 0 & 0 & 0 & 0 & 0 & 0 & 0 & 0 & 0 & 0 & 0 \\
\hline 224.485 & 0 & 0 & 0 & 0 & 0 & 0 & 0 & 0 & 0 & 0 & 0 & 0 \\
\hline 224.385 & 0 & 0 & 0 & 0 & 0 & 0 & 0 & 0 & 0 & 0 & 0 & 0 \\
\hline 224.285 & 0 & 0 & 0 & 0 & 0 & 0 & 0 & 0 & 0 & 0 & 0 & 0 \\
\hline 224.185 & 0 & 0 & 0 & 0 & 0 & 0 & 0 & 0 & 0 & 0 & 0 & 0 \\
\hline 224.085 & 0 & 0 & 0 & 0 & 0 & 0 & 0 & 0 & 0 & 0 & 0 & 0 \\
\hline 223.985 & 0 & 0 & 0 & 0 & 0 & 0 & 0 & 0 & 0 & 0 & 0 & 0 \\
\hline 223.885 & 0 & 0 & 0 & 0 & 0 & 0 & 0 & 0 & 0 & 0 & 0 & 0 \\
\hline 223.785 & 0 & 0 & 0 & 0 & 0 & 0 & 0 & 0 & 0 & 0 & 0 & 0 \\
\hline 223.685 & 0 & 0 & 0 & 0 & 0 & 0 & 0 & 0 & 0 & 0 & 0 & 0 \\
\hline 223.585 & 0 & 0 & 0 & 0 & 0 & 0 & 0 & 0 & 0 & 0 & 0 & 0 \\
\hline 223.485 & 0 & 0 & 0 & 0 & 0 & 0 & 0 & 0 & 0 & 0 & 0 & 0 \\
\hline 223.385 & 0 & 0 & 0 & 0 & 0 & 0 & 0 & 0 & 0 & 0 & 0 & 0 \\
\hline 223.285 & 0 & 0 & 0 & 0 & 0 & 0 & 0 & 0 & 0 & 0 & 0 & 0 \\
\hline 223.185 & 0 & 0 & 0 & 0 & 0 & 0 & 0 & 0 & 0 & 0 & 0 & 0 \\
\hline 223.085 & 0 & 0 & 0 & 0 & 0 & 0 & 0 & 0 & 0 & 0 & 0 & 0 \\
\hline 222.985 & 0 & 0 & 0 & 0 & 0 & 0 & 0 & 0 & 0 & 0 & 0 & 0 \\
\hline 222.885 & 0 & 0 & 0 & 0 & 0 & 0 & 0 & 0 & 0 & 0 & 0 & 0 \\
\hline
\end{tabular}




\begin{tabular}{|c|c|c|c|c|c|c|c|c|c|c|c|c|}
\hline \multirow{2}{*}{$\begin{array}{c}\text { Interval } \\
\text { (Ma) }\end{array}$} & \multicolumn{3}{|c|}{ Acaenasuchus } & \multicolumn{3}{|c|}{ Trilophosaurus } & \multicolumn{3}{|c|}{ Calyptosuchus } & \multicolumn{3}{|c|}{ Placerias } \\
\hline & Solow & $\begin{array}{l}\text { Alroy } \\
\text { (NU) }\end{array}$ & $\begin{array}{l}\text { Alroy } \\
\text { (U) }\end{array}$ & Solow & $\begin{array}{l}\text { Alroy } \\
\text { (NU) }\end{array}$ & $\begin{array}{l}\text { Alroy } \\
\text { (U) }\end{array}$ & Solow & $\begin{array}{l}\text { Alroy } \\
\text { (NU) }\end{array}$ & $\begin{array}{l}\text { Alroy } \\
\text { (U) }\end{array}$ & Solow & $\begin{array}{l}\text { Alroy } \\
\text { (NU) }\end{array}$ & $\begin{array}{l}\text { Alroy } \\
\text { (U) }\end{array}$ \\
\hline 222.785 & 0 & 0 & 0 & 0 & 0 & 0 & 0 & 0 & 0 & 0 & 0 & 0 \\
\hline 222.685 & 0 & 0 & 0 & 0 & 0 & 0 & 0 & 0 & 0 & 0 & 0 & 0 \\
\hline 222.585 & 0 & 0 & 0 & 0 & 0 & 0 & 0 & 0 & 0 & 0 & 0 & 0 \\
\hline 222.485 & 0 & 0 & 0 & 0 & 0 & 0 & 0 & 0 & 0 & 0 & 0 & 0 \\
\hline 222.385 & 0 & 0 & 0 & 0 & 0 & 0 & 0 & 0 & 0 & 0 & 0 & 0 \\
\hline 222.285 & 0 & 0 & 0 & 0 & 0 & 0 & 0 & 0 & 0 & 0 & 0 & 0 \\
\hline 222.185 & 0 & 0 & 0 & 0 & 0 & 0 & 0 & 0 & 0 & 0 & 0 & 0 \\
\hline 222.085 & 0 & 0 & 0 & 0 & 0 & 0 & 0 & 0 & 0 & 0 & 0 & 0 \\
\hline 221.985 & 0 & 0 & 0 & 0 & 0 & 0 & 0 & 0 & 0 & 0 & 0 & 0 \\
\hline 221.885 & 0 & 0 & 0 & 0 & 0 & 0 & 0 & 0 & 0 & 0 & 0 & 0 \\
\hline 221.785 & 0 & 0 & 0 & 0 & 0 & 0 & 0 & 0 & 0 & 0 & 0 & 0 \\
\hline 221.685 & 0 & 0 & 0 & 0 & 0 & 0 & 0 & 0 & 0 & 0 & 0 & 0 \\
\hline 221.585 & 0 & 0 & 0 & 0 & 0 & 0 & 0 & 0 & 0 & 0 & 0 & 0 \\
\hline 221.485 & 0 & 0 & 0 & 0 & 0 & 0 & 0 & 0 & 0 & 0 & 0 & 0 \\
\hline 221.385 & 0 & 0 & 0 & 0 & 0 & 0 & 0 & 0 & 0 & 0 & 0 & 0 \\
\hline 221.285 & 0 & 0 & 0 & 0 & 0 & 0 & 0 & 0 & 0 & 0 & 0 & 0 \\
\hline 221.185 & 0 & 0 & 0 & 0 & 0 & 0 & 0 & 0 & 0 & 0 & 0 & 0 \\
\hline 221.085 & 0 & 0 & 0 & 0 & 0 & 0 & 0 & 0 & 0 & 0 & 0 & 0 \\
\hline 220.985 & 0 & 0 & 0 & 0 & 0 & 0 & 0 & 0 & 0 & 0 & 0 & 0 \\
\hline 220.885 & 0 & 0 & 0 & 0 & 0 & 0 & 0 & 0 & 0 & 0 & 0 & 0 \\
\hline 220.785 & 0 & 0 & 0 & 0 & 0 & 0 & 0 & 0 & 0 & 0 & 0 & 0 \\
\hline 220.685 & 0 & 0 & 0 & 0 & 0 & 0 & 0 & 0 & 0 & 0 & 0 & 0 \\
\hline 220.585 & 0 & 0 & 0 & 0 & 0 & 0 & 0 & 0 & 0 & 0 & 0 & 0 \\
\hline 220.485 & 0 & 0 & 0 & 0 & 0 & 0 & 0 & 0 & 0 & 0 & 0 & 0 \\
\hline 220.385 & 0 & 0 & 0 & 0 & 0 & 0 & 0 & 0 & 0 & 0 & 0 & 0 \\
\hline
\end{tabular}




\begin{tabular}{|c|c|c|c|c|c|c|c|c|c|c|c|c|}
\hline \multirow{2}{*}{$\begin{array}{c}\text { Interval } \\
\text { (Ma) }\end{array}$} & \multicolumn{3}{|c|}{ Acaenasuchus } & \multicolumn{3}{|c|}{$\underline{\text { Trilophosaurus }}$} & \multicolumn{3}{|c|}{ Calyptosuchus } & \multicolumn{3}{|c|}{ Placerias } \\
\hline & Solow & $\begin{array}{l}\text { Alroy } \\
\text { (NU) }\end{array}$ & $\begin{array}{l}\text { Alroy } \\
\text { (U) }\end{array}$ & Solow & $\begin{array}{l}\text { Alroy } \\
\text { (NU) }\end{array}$ & $\begin{array}{l}\text { Alroy } \\
\text { (U) }\end{array}$ & Solow & $\begin{array}{l}\text { Alroy } \\
\text { (NU) }\end{array}$ & $\begin{array}{l}\text { Alroy } \\
\text { (U) }\end{array}$ & Solow & $\begin{array}{l}\text { Alroy } \\
\text { (NU) }\end{array}$ & $\begin{array}{l}\text { Alroy } \\
\text { (U) }\end{array}$ \\
\hline 220.285 & 0 & 0 & 0 & 0 & 0 & 0 & 0 & 0 & 0 & 0 & 0 & 0 \\
\hline 220.185 & 0 & 0 & 0 & 0 & 0 & 0 & 0 & 0 & 0 & 0 & 0 & 0 \\
\hline 220.085 & 0 & 0 & 0 & 0 & 0 & 0 & 0 & 0 & 0 & 0 & 0 & 0 \\
\hline 219.985 & $2.11 \mathrm{E}-05$ & 2.09E-05 & $1.20 \mathrm{E}-05$ & 0 & 0 & 0 & 0 & 0 & 0 & 0 & 0 & 0 \\
\hline 219.885 & 0.000187 & 0.000181 & 0.000104 & 0 & 0 & 0 & 0 & 0 & 0 & 0 & 0 & 0 \\
\hline 219.785 & 0.000207 & 0.000197 & 0.000114 & 0 & 0 & 0 & $1.98 \mathrm{E}-05$ & $1.69 \mathrm{E}-05$ & 8.91E-06 & 0 & 0 & 0 \\
\hline 219.685 & 0.000368 & 0.000343 & 0.000199 & 0 & 0 & 0 & 4.04E-05 & 3.33E-05 & $1.76 \mathrm{E}-05$ & 0 & 0 & 0 \\
\hline 219.585 & 0.001105 & 0.00104 & 0.0006 & 0 & 0 & 0 & 8.09E-05 & $6.77 \mathrm{E}-05$ & 3.59E-05 & 0 & 0 & 0 \\
\hline 219.485 & 0.004326 & 0.00415 & 0.002399 & $5.70 \mathrm{E}-05$ & 4.64E-05 & $2.54 \mathrm{E}-05$ & 0.000104 & $8.75 \mathrm{E}-05$ & 4.67E-05 & 4.46E-05 & 4.90E-05 & $3.10 E-05$ \\
\hline 219.385 & 0.010628 & 0.010483 & 0.006125 & $9.52 E-05$ & $7.56 \mathrm{E}-05$ & 4.15E-05 & 0.000145 & 0.000124 & $6.67 \mathrm{E}-05$ & $6.72 \mathrm{E}-05$ & 7.06E-05 & 4.49E-05 \\
\hline 219.285 & 0.01598 & 0.015453 & 0.009026 & 0.000337 & 0.000274 & 0.00015 & 0.00015 & 0.00013 & $7.06 \mathrm{E}-05$ & 0.00024 & 0.000251 & 0.000159 \\
\hline 219.185 & 0.018792 & 0.017988 & 0.010561 & 0.002044 & 0.001671 & 0.000914 & 0.000246 & 0.000209 & 0.000113 & 0.000286 & 0.000298 & 0.000191 \\
\hline 219.085 & 0.019614 & 0.018651 & 0.011042 & 0.00707 & 0.005797 & 0.003174 & 0.000346 & 0.000291 & 0.000157 & 0.00029 & 0.000302 & 0.000195 \\
\hline 218.985 & 0.019861 & 0.018799 & 0.011238 & 0.013433 & 0.010966 & 0.006018 & 0.000714 & 0.000599 & 0.000321 & 0.000292 & 0.000304 & 0.000199 \\
\hline 218.885 & 0.02002 & 0.018884 & 0.011405 & 0.016988 & 0.013877 & 0.007655 & 0.001966 & 0.00165 & 0.000876 & 0.000295 & 0.000307 & 0.000203 \\
\hline 218.785 & 0.020126 & 0.018938 & 0.01156 & 0.018004 & 0.014725 & 0.008187 & 0.004443 & 0.003696 & 0.001956 & 0.000297 & 0.000308 & 0.000207 \\
\hline 218.685 & 0.020198 & 0.018976 & 0.011713 & 0.018672 & 0.015305 & 0.008582 & 0.00713 & 0.005947 & 0.003155 & 0.000298 & 0.00031 & 0.000211 \\
\hline 218.585 & 0.020234 & 0.018999 & 0.011863 & 0.019272 & 0.015848 & 0.008968 & 0.010333 & 0.008626 & 0.004591 & 0.000299 & 0.000311 & 0.000215 \\
\hline 218.485 & 0.020238 & 0.019005 & 0.01201 & 0.019719 & 0.016291 & 0.009311 & 0.013386 & 0.01123 & 0.006006 & 0.000299 & 0.000311 & 0.000218 \\
\hline 218.385 & 0.020208 & 0.018995 & 0.012154 & 0.020124 & 0.016717 & 0.009655 & 0.016271 & 0.013728 & 0.007388 & 0.000299 & 0.000312 & 0.000222 \\
\hline 218.285 & 0.020147 & 0.018968 & 0.012293 & 0.020486 & 0.017125 & 0.010001 & 0.018559 & 0.015797 & 0.008573 & 0.000299 & 0.000311 & 0.000225 \\
\hline 218.185 & 0.020056 & 0.018924 & 0.012427 & 0.020709 & 0.017442 & 0.01031 & 0.020019 & 0.017239 & 0.009458 & 0.000298 & 0.00031 & 0.000228 \\
\hline 218.085 & 0.019935 & 0.018863 & 0.012557 & 0.020901 & 0.017751 & 0.010626 & 0.021017 & 0.018353 & 0.010204 & 0.000296 & 0.000309 & 0.000231 \\
\hline 217.985 & 0.019787 & 0.018785 & 0.012682 & 0.021047 & 0.018037 & 0.010942 & 0.021861 & 0.019387 & 0.01094 & 0.000294 & 0.000307 & 0.000234 \\
\hline 217.885 & 0.019614 & 0.01869 & 0.012801 & 0.021131 & 0.018287 & 0.01125 & 0.022586 & 0.020359 & 0.011682 & 0.000327 & 0.000346 & 0.000262 \\
\hline
\end{tabular}




\begin{tabular}{|c|c|c|c|c|c|c|c|c|c|c|c|c|}
\hline \multirow{2}{*}{$\begin{array}{c}\text { Interval } \\
\text { (Ma) }\end{array}$} & \multicolumn{3}{|c|}{ Acaenasuchus } & \multicolumn{3}{|c|}{ Trilophosaurus } & \multicolumn{3}{|c|}{ Calyptosuchus } & \multicolumn{3}{|c|}{ Placerias } \\
\hline & Solow & $\begin{array}{l}\text { Alroy } \\
\text { (NU) }\end{array}$ & $\begin{array}{l}\text { Alroy } \\
(U)\end{array}$ & Solow & $\begin{array}{l}\text { Alroy } \\
\text { (NU) }\end{array}$ & $\begin{array}{l}\text { Alroy } \\
(U)\end{array}$ & Solow & $\begin{array}{l}\text { Alroy } \\
\text { (NU) }\end{array}$ & $\begin{array}{l}\text { Alroy } \\
(U)\end{array}$ & Solow & $\begin{array}{l}\text { Alroy } \\
\text { (NU) }\end{array}$ & $\begin{array}{l}\text { Alroy } \\
\text { (U) }\end{array}$ \\
\hline 217.785 & 0.019416 & 0.018579 & 0.012914 & 0.021168 & 0.018512 & 0.011557 & 0.023238 & 0.021306 & 0.012449 & 0.000446 & 0.000457 & 0.000335 \\
\hline 217.685 & 0.019195 & 0.018451 & 0.013021 & 0.021161 & 0.01871 & 0.01186 & 0.023811 & 0.022215 & 0.013239 & 0.000686 & 0.00067 & 0.000472 \\
\hline 217.585 & 0.018954 & 0.018307 & 0.013121 & 0.021109 & 0.01888 & 0.01216 & 0.024297 & 0.023073 & 0.014047 & 0.001113 & 0.001059 & 0.000718 \\
\hline 217.485 & 0.018694 & 0.018148 & 0.013215 & 0.021014 & 0.01902 & 0.012455 & 0.024691 & 0.023867 & 0.014867 & 0.001511 & 0.001417 & 0.000948 \\
\hline 217.385 & 0.018417 & 0.017973 & 0.013301 & 0.020879 & 0.01913 & 0.012744 & 0.025004 & 0.024596 & 0.015699 & 0.001961 & 846 & 0.001223 \\
\hline 217.285 & 0.018124 & 0.017784 & 0.013379 & 0.020706 & 0.019209 & 0.013026 & 0.025206 & 0.025225 & 0.016523 & 0.002996 & 0.002787 & 0.001817 \\
\hline 217.185 & 0.017817 & 0.017581 & 0.01345 & 0.020496 & 0.0 & 0.0133 & 0.025309 & 0.025 & 0.017337 & 0.005413 & 0.004956 & 0.003175 \\
\hline 217.085 & 0.017498 & 0.017365 & 0.013513 & & & 0.013564 & 0.025315 & 0.026178 & 0.018133 & 0.008829 & 0.008033 & 0.005103 \\
\hline 216.985 & 0.017169 & 0.017136 & 0.013568 & 0.019977 & 0.0192 & 0.013818 & 0.025226 & 0.026487 & 0.018901 & 0.01207 & 0.010923 & 0.006929 \\
\hline 216.885 & 0.016831 & 0.016895 & 0.013615 & 0.019673 & 9211 & 0.014061 & 0.025045 & 0.026676 & 0.019631 & 0.013964 & 0.012634 & 0.008043 \\
\hline 216.785 & 0.016485 & 0.016643 & 0.013653 & 0.019343 & 0.019132 & 0.014292 & 0.024778 & 0.026743 & 0.020315 & 0.01457 & 3207 & 0.008466 \\
\hline 216.685 & 0.016133 & 0.01638 & 0.013682 & 0.018991 & $0.019 c$ & 0.014 & 0.02 & 0.0 & 0.020 & 0.0 & 622 & 0.008799 \\
\hline 216.585 & 0.015776 & 0.016108 & 0.013702 & 0.018617 & 0.018884 & 0.014712 & 0.02401 & 0.026518 & 0.021507 & 0.015438 & 0.014079 & 0.009167 \\
\hline 216.485 & 0.015416 & 0.015827 & 0.013714 & 0.018226 & 0.018715 & 0.014899 & 0.023523 & 0.026231 & 0.021998 & 0.01576 & 0.01442 & 0.009471 \\
\hline 216.385 & 0.015054 & 0.015539 & 0.013717 & 0.01782 & 0.018519 & 0.01507 & 0.022991 & 0.025847 & 0.022417 & 0.016078 & 0.014765 & 0.009785 \\
\hline 216.285 & 0.014691 & 0.015243 & 0.01371 & 0.017401 & 0.018296 & 0.015224 & 0.022396 & 0.025355 & 0.022747 & 0.016406 & 0.015122 & 0.010116 \\
\hline 216.185 & 0.014327 & 0.014941 & 0.013695 & 0.016972 & 0.018048 & 0.01536 & 0.02176 & 0.024774 & 0.022989 & 0.016658 & 0.015426 & 0.010422 \\
\hline 216.085 & 0.013965 & 0.014634 & 0.013671 & 0.016535 & 0.017777 & 0.015478 & 0.021088 & 0.024113 & 0.023142 & 0.016987 & 0.0158 & 0.01078 \\
\hline 215.985 & 0.013604 & 0.014322 & 0.013638 & 0.016092 & 0.017483 & 0.015577 & 0.020391 & 0.023385 & 0.023204 & 0.017213 & 0.016081 & 0.01109 \\
\hline 215.885 & 0.013245 & 0.014007 & 0.013596 & 0.015645 & 0.017169 & 0.015656 & 0.019673 & 0.022599 & 0.023176 & 0.017419 & 0.016352 & 0.011402 \\
\hline 215.785 & 0.01289 & 0.013688 & 0.013545 & 0.015196 & 0.016836 & 0.015715 & 0.018942 & 0.021769 & 0.02306 & 0.017576 & 0.016586 & 0.0117 \\
\hline 215.685 & 0.012539 & 0.013367 & 0.013486 & 0.014747 & 0.016487 & 0.015754 & 0.018204 & 0.020903 & 0.022861 & 0.017711 & 0.016801 & 0.011995 \\
\hline 215.585 & 0.012192 & 0.013044 & 0.013418 & 0.014299 & 0.016122 & 0.015773 & 0.017464 & 0.020014 & 0.022582 & 0.017838 & 0.017014 & 0.012298 \\
\hline 215.485 & 0.011849 & 0.012721 & 0.013342 & 0.013854 & 0.015745 & 0.015771 & 0.016728 & 0.019109 & 0.022229 & 0.017931 & 0.017193 & 0.012587 \\
\hline 215.385 & 0.011513 & 0.012397 & 0.013258 & 0.013413 & 0.015357 & 0.015749 & 0.015999 & 0.018199 & 0.021809 & 0.018016 & 0.017365 & 0.01288 \\
\hline
\end{tabular}




\begin{tabular}{|c|c|c|c|c|c|c|c|c|c|c|c|c|}
\hline \multirow{2}{*}{$\begin{array}{c}\text { Interval } \\
\text { (Ma) }\end{array}$} & \multicolumn{3}{|c|}{ Acaenasuchus } & \multicolumn{3}{|c|}{ Trilophosaurus } & \multicolumn{3}{|c|}{ Calyptosuchus } & \multicolumn{3}{|c|}{ Placerias } \\
\hline & Solow & $\begin{array}{l}\text { Alroy } \\
\text { (NU) }\end{array}$ & $\begin{array}{l}\text { Alroy } \\
(U)\end{array}$ & Solow & $\begin{array}{l}\text { Alroy } \\
\text { (NU) }\end{array}$ & $\begin{array}{l}\text { Alroy } \\
(U)\end{array}$ & Solow & $\begin{array}{l}\text { Alroy } \\
\text { (NU) }\end{array}$ & $\begin{array}{l}\text { Alroy } \\
(U)\end{array}$ & Solow & $\begin{array}{l}\text { Alroy } \\
\text { (NU) }\end{array}$ & $\begin{array}{l}\text { Alroy } \\
\text { (U) }\end{array}$ \\
\hline 215.285 & 0.011182 & 0.012074 & 0.013166 & 0.012976 & 0.014959 & 0.015707 & 0.015282 & 0.01729 & 0.021328 & 0.018054 & 0.017499 & 0.013158 \\
\hline 215.185 & 0.010857 & 0.011752 & 0.013066 & 0.012547 & 0.014554 & 0.015645 & 0.014578 & 0.01639 & 0.020794 & 0.01806 & 0.017602 & 0.013423 \\
\hline 215.085 & 0.010538 & 0.011431 & 0.012958 & 0.012124 & 0.014142 & 0.015563 & 0.013892 & 0.015505 & 0.020215 & 0.018095 & 0.017726 & 0.013707 \\
\hline 214.985 & 0.010226 & 0.011113 & 0.012844 & 0.011709 & 0.013727 & 0.015462 & 0.013224 & 0.014639 & 0.019597 & 0.018062 & 0.017789 & 0.01396 \\
\hline 214.885 & 0.00992 & 0.010797 & 0.012722 & 0.011302 & 0.013309 & 0.015343 & 0.012577 & 0.013797 & 0.018949 & 0.01801 & 0.017831 & 0.014206 \\
\hline 214.785 & 0.009622 & 0.010485 & 0.012594 & 0.010904 & 0.01289 & 0.015206 & 0.011952 & 0.012983 & 0.018276 & 0.017952 & 0.017863 & 0.014449 \\
\hline 214.685 & 0.009331 & 0.010175 & 0.01246 & 0.010516 & 0.012471 & 0.015052 & 0.01135 & 0.012 & 0.017586 & 0.017841 & 0.017847 & 0.014668 \\
\hline 214.585 & 0.009046 & 0.00987 & 0.012319 & 0.010138 & 0.012053 & 0.014881 & 0.010772 & 0.011446 & 0.016885 & 0.017713 & 0.01781 & 0.014877 \\
\hline 214.485 & 0.008769 & 0.009569 & 0.012173 & 0.009769 & 0.011638 & 0.014695 & 0.010217 & 0.010726 & 0.016178 & 0.01757 & 0.017754 & 0.015076 \\
\hline 214.385 & 0.008499 & 0.009273 & 0.012022 & 0.009411 & 0.011227 & 0.014494 & 0.009686 & 0.010 & 0.015471 & 0.0174 & 1767 & 0.01526 \\
\hline 214.285 & 0.008237 & 0.008981 & 0.011865 & 0.009063 & 0.010821 & 0.014279 & 0.009178 & 389 & 0.014768 & 0.017262 & 0.017605 & 0.015456 \\
\hline 214.185 & 0.007981 & 0.008695 & 0.011704 & 0.008726 & 0.0 & 0.014052 & 0.008694 & & 0.014073 & 0.01 & 485 & 0.015618 \\
\hline 214.085 & 0.007733 & 0.008414 & 0.011538 & 0.008399 & 0.010027 & 0.013813 & 0.008233 & 0.008 & 0.01339 & 0.016857 & 0.017346 & 0.015767 \\
\hline 213.985 & 0.007491 & 0.008139 & 0.011368 & 0.008083 & 0.009641 & 0.013563 & 0.007795 & 0.007636 & 0.012722 & 0.016638 & 0.017192 & 0.015905 \\
\hline 213.885 & 0.007257 & 0.007869 & 0.011195 & 0.007777 & 0.009262 & 0.013304 & 0.007378 & 0.007118 & 0.01207 & 0.016408 & 0.01702 & 0.01603 \\
\hline 213.785 & 0.007029 & 0.007605 & 0.011018 & 0.007482 & 0.008892 & 0.013036 & 0.006982 & 0.006631 & 0.011438 & 0.016169 & 0.016834 & 0.016142 \\
\hline 213.685 & 0.006808 & 0.007348 & 0.010839 & 0.007196 & 0.008531 & 0.01276 & 0.006607 & 0.006173 & 0.010826 & 0.015922 & 0.016633 & 0.01624 \\
\hline 213.585 & 0.006594 & 0.007096 & 0.010656 & 0.006921 & 0.008179 & 0.012479 & & & 0.010236 & 0.015667 & 0.016419 & 0.016325 \\
\hline 213.485 & 0.006386 & 0.00685 & 0.010472 & 0.006655 & 0.007836 & 0.012191 & 0.005915 & 0.005344 & 0.009669 & 0.015406 & 0.016192 & 0.016395 \\
\hline 213.385 & 0.006185 & 0.006611 & 0.010285 & 0.0064 & 0.007504 & 0.011899 & 0.005597 & 0.004969 & 0.009125 & 0.015139 & 0.015952 & 0.016452 \\
\hline 213.285 & 0.00599 & 0.006378 & 0.010096 & 0.006153 & 0.007182 & 0.011603 & 0.005295 & 0.00462 & 0.008605 & 0.014867 & 0.015703 & 0.016494 \\
\hline 213.185 & 0.005801 & 0.006151 & 0.009907 & 0.005916 & 0.006869 & 0.011305 & 0.00501 & 0.004293 & 0.008108 & 0.014591 & 0.015443 & 0.016523 \\
\hline 213.085 & 0.005618 & 0.005931 & 0.009716 & 0.005687 & 0.006567 & 0.011005 & 0.004741 & 0.003989 & 0.007634 & 0.014312 & 0.015174 & 0.016537 \\
\hline 212.985 & 0.005441 & 0.005716 & 0.009524 & 0.005468 & 0.006275 & 0.010704 & 0.004487 & 0.003706 & 0.007184 & 0.01403 & 0.014897 & 0.016536 \\
\hline 212.885 & 0.00527 & 0.005508 & 0.009332 & 0.005257 & 0.005994 & 0.010403 & 0.004247 & 0.003442 & 0.006757 & 0.013746 & 0.014613 & 0.016522 \\
\hline
\end{tabular}




\begin{tabular}{|c|c|c|c|c|c|c|c|c|c|c|c|c|}
\hline \multirow{2}{*}{$\begin{array}{c}\text { Interval } \\
\text { (Ma) }\end{array}$} & \multicolumn{3}{|c|}{ Acaenasuchus } & \multicolumn{3}{|c|}{ Trilophosaurus } & \multicolumn{3}{|c|}{ Calyptosuchus } & \multicolumn{3}{|c|}{ Placerias } \\
\hline & Solow & $\begin{array}{l}\text { Alroy } \\
\text { (NU) }\end{array}$ & $\begin{array}{l}\text { Alroy } \\
\text { (U) }\end{array}$ & Solow & $\begin{array}{l}\text { Alroy } \\
\text { (NU) }\end{array}$ & $\begin{array}{l}\text { Alroy } \\
\text { (U) }\end{array}$ & Solow & $\begin{array}{l}\text { Alroy } \\
\text { (NU) }\end{array}$ & $\begin{array}{l}\text { Alroy } \\
\text { (U) }\end{array}$ & Solow & $\begin{array}{l}\text { Alroy } \\
\text { (NU) }\end{array}$ & $\begin{array}{l}\text { Alroy } \\
\text { (U) }\end{array}$ \\
\hline 212.785 & 0.005104 & 0.005306 & 0.00914 & 0.005054 & 0.005722 & 0.010103 & 0.00402 & 0.003197 & 0.006352 & 0.013461 & 0.014323 & 0.016493 \\
\hline 212.685 & 0.004944 & 0.00511 & 0.008947 & 0.004859 & 0.00546 & 0.009803 & 0.003806 & 0.002969 & 0.005968 & 0.013176 & 0.014027 & 0.016451 \\
\hline 212.585 & 0.004788 & 0.00492 & 0.008755 & 0.004671 & 0.005209 & 0.009506 & 0.003604 & 0.002757 & 0.005606 & 0.01289 & 0.013727 & 0.016395 \\
\hline 212.485 & 0.004638 & 0.004736 & 0.008564 & 0.004491 & 0.004967 & 0.009211 & 0.003413 & 0.002561 & 0.005263 & 0.012605 & 0.013424 & 0.016326 \\
\hline 212.385 & 0.004493 & 0.004558 & 0.008373 & 0.004318 & 0.004735 & 0.008919 & 0.003233 & 0.002378 & 0.00494 & 0.012321 & 0.013117 & 0.016244 \\
\hline 212.285 & 0.004353 & 0.004385 & 0.008183 & 0.004152 & 0.004512 & 0.008631 & 0.003063 & 0.002208 & 0.004636 & 0.012038 & 0.012809 & 0.016149 \\
\hline 212.185 & 0.004217 & 0.004219 & 0.007995 & 0.003993 & 0.004298 & 0.008347 & 0.002903 & 0.002051 & 0.004349 & 0.011757 & 0.012499 & 0.016041 \\
\hline 212.085 & 0.004086 & 0.004057 & 0.007807 & 0.00384 & 0.004093 & 0.008068 & 0.002751 & 0.00 & 0.004079 & 0.011 & 2189 & 0.015922 \\
\hline 211.985 & 0.003959 & 0.003902 & 0.007622 & 0.003693 & 0.003897 & 0.007793 & 0.002608 & 0.00177 & 0.003826 & 0.011203 & 0.011878 & 0.015792 \\
\hline 211.885 & 0.003837 & 0.003751 & 0.007438 & 0.003552 & 0.003709 & 0.007524 & 0.002474 & 0.001644 & 0.003587 & 0.01093 & 0.011569 & 0.01565 \\
\hline 211.785 & 0.003718 & 0.003606 & 0.007256 & 0.003417 & 0.00353 & 0.007259 & 0.002346 & 0.001527 & 0.003363 & 0.01066 & 0.01126 & 0.015498 \\
\hline 211.685 & 0.003604 & 0.003465 & 0.007075 & 0.003288 & 0.003358 & 0.007001 & 0.002226 & 0.001 & 0.003153 & 0.010394 & 0.010954 & 0.015336 \\
\hline 211.585 & 0.003493 & 0.00333 & 0.006898 & 0.003163 & 0.0031 & 0.006748 & 0.002113 & & 0.002956 & 0.010132 & & 0.015165 \\
\hline 211.485 & 0.003386 & 0.003199 & 0.006722 & 0.003044 & 0.003037 & 0.006502 & 0.002006 & 0.001226 & 0.002771 & 0.009873 & 0.010348 & 0.014985 \\
\hline 211.385 & 0.003282 & 0.003074 & 0.006549 & 0.00293 & 0.002888 & 0.006262 & 0.001904 & 0.00114 & 0.002597 & 0.009619 & 0.01005 & 0.014796 \\
\hline 211.285 & 0.003183 & 0.002952 & 0.006378 & 0.00282 & 0.002745 & 0.006027 & 0.001809 & 0.00106 & 0.002435 & 0.009369 & 0.009756 & 0.0146 \\
\hline 211.185 & 0.003086 & 0.002835 & 0.00621 & 0.002714 & 0.002609 & 0.0058 & 0.001718 & 0.000986 & 0.002282 & 0.009123 & 0.009465 & 0.014397 \\
\hline 211.085 & 0.002993 & 0.002723 & 0.006045 & 0.002613 & 0.00248 & 0.005579 & 0.001633 & 0.000917 & 0.00214 & 0.008882 & 0.009179 & 0.014187 \\
\hline 210.985 & 0.002902 & 0.002614 & 0.005882 & 0.002516 & 0.002356 & 0.005364 & 0.001552 & 0.000853 & 0.002006 & 0.008646 & 0.008898 & 0.013971 \\
\hline 210.885 & 0.002815 & 0.00251 & 0.005722 & 0.002423 & 0.002238 & 0.005155 & 0.001476 & 0.000794 & 0.001881 & 0.008414 & 0.008621 & 0.013749 \\
\hline 210.785 & 0.002731 & 0.002409 & 0.005565 & 0.002334 & 0.002126 & 0.004953 & 0.001404 & 0.000739 & 0.001763 & 0.008187 & 0.008349 & 0.013523 \\
\hline 210.685 & 0.002649 & 0.002313 & 0.005411 & 0.002248 & 0.00202 & 0.004758 & 0.001336 & 0.000688 & 0.001654 & 0.007965 & 0.008083 & 0.013292 \\
\hline 210.585 & 0.002571 & 0.00222 & 0.00526 & 0.002166 & 0.001918 & 0.004568 & 0.001271 & & 0.001551 & 0.007748 & 0.007822 & 0.013057 \\
\hline 210.485 & 0.002495 & 0.00213 & 0.005112 & 0.002087 & 0.001822 & 0.004385 & 0.00121 & 0.000597 & 0.001455 & 0.007535 & 0.007567 & 0.012819 \\
\hline 210.385 & 0.002421 & 0.002044 & 0.004966 & 0.002011 & 0.00173 & 0.004209 & 0.001152 & 0.000556 & 0.001365 & 0.007328 & 0.007317 & 0.012578 \\
\hline
\end{tabular}




\begin{tabular}{|c|c|c|c|c|c|c|c|c|c|c|c|c|}
\hline \multirow{2}{*}{$\begin{array}{c}\text { Interval } \\
\text { (Ma) }\end{array}$} & \multicolumn{3}{|c|}{ Acaenasuchus } & \multicolumn{3}{|c|}{ Trilophosaurus } & \multicolumn{3}{|c|}{ Calyptosuchus } & \multicolumn{3}{|c|}{ Placerias } \\
\hline & Solow & $\begin{array}{l}\text { Alroy } \\
\text { (NU) }\end{array}$ & $\begin{array}{l}\text { Alroy } \\
\text { (U) }\end{array}$ & Solow & $\begin{array}{l}\text { Alroy } \\
\text { (NU) }\end{array}$ & $\begin{array}{l}\text { Alroy } \\
\text { (U) }\end{array}$ & Solow & $\begin{array}{l}\text { Alroy } \\
\text { (NU) }\end{array}$ & $\begin{array}{l}\text { Alroy } \\
\text { (U) }\end{array}$ & Solow & $\begin{array}{l}\text { Alroy } \\
\text { (NU) }\end{array}$ & $\begin{array}{l}\text { Alroy } \\
\text { (U) }\end{array}$ \\
\hline 210.285 & 0.00235 & 0.001962 & 0.004824 & 0.001939 & 0.001643 & 0.004038 & 0.001098 & 0.000518 & 0.00128 & 0.007125 & 0.007073 & 0.012335 \\
\hline 210.185 & 0.002281 & 0.001882 & 0.004685 & 0.001869 & 0.001559 & 0.003873 & 0.001046 & 0.000483 & 0.001202 & 0.006927 & 0.006835 & 0.01209 \\
\hline 210.085 & 0.002214 & 0.001806 & 0.004549 & 0.001802 & 0.001481 & 0.003714 & 0.000997 & 0.00045 & 0.001128 & 0.006734 & 0.006603 & 0.011843 \\
\hline 209.985 & 0.00215 & 0.001733 & 0.004416 & 0.001738 & 0.001406 & 0.003561 & 0.00095 & 0.00042 & 0.001059 & 0.006546 & 0.006377 & 0.011596 \\
\hline
\end{tabular}


TABLE S10. AVERAGE POSTERIOR EXTINCTION AND ORIGINATION PROBABILITIES GENERATED FOR DESMATOSUCHUS, SMILOSUCHUS, TYPOTHORAX, AND PARATYPOTHORAX BY ALROY (2014) AND SOLOW (2016) METHODS. "NO UNDERSAMPLING" AND “UNDERSAMPLING" DENTOED RESPECTIVELY BY "NU” AND "U”.

\begin{tabular}{|c|c|c|c|c|c|c|c|c|c|c|c|c|}
\hline \multirow{2}{*}{$\begin{array}{c}\text { Interval } \\
\text { (Ma) }\end{array}$} & \multicolumn{3}{|c|}{ Desmatosuchus } & \multicolumn{3}{|c|}{$\underline{\text { Smilosuchus }}$} & \multicolumn{3}{|c|}{ Typothorax } & \multicolumn{3}{|c|}{ Paratypothorax } \\
\hline & Solow & $\begin{array}{l}\text { Alroy } \\
\text { (NU) }\end{array}$ & $\begin{array}{l}\text { Alroy } \\
\text { (U) }\end{array}$ & Solow & $\begin{array}{l}\text { Alroy } \\
\text { (NU) }\end{array}$ & $\begin{array}{l}\text { Alroy } \\
\text { (U) }\end{array}$ & Solow & $\begin{array}{l}\text { Alroy } \\
\text { (NU) }\end{array}$ & $\begin{array}{l}\text { Alroy } \\
\text { (U) }\end{array}$ & Solow & $\begin{array}{l}\text { Alroy } \\
\text { (NU) }\end{array}$ & $\begin{array}{l}\text { Alroy } \\
\text { (U) }\end{array}$ \\
\hline 225.185 & 0 & 0 & 0 & 0 & 0 & 0 & 0.000521 & 0.000247 & 0.000552 & 0.005568 & 0.004483 & 0.009239 \\
\hline 225.085 & 0 & 0 & 0 & 0 & 0 & 0 & 0.000592 & 0.000293 & 0.000653 & 0.005809 & 0.00476 & 0.009675 \\
\hline 224.985 & 0 & 0 & 0 & 0 & 0 & 0 & 0.000673 & 0.000348 & 0.000771 & 0.006059 & 0.005052 & 0.010123 \\
\hline 224.885 & 0 & 0 & 0 & 0 & 0 & 0 & 0.000765 & 0.000414 & 0.000911 & 0.00632 & 0.00536 & 0.010582 \\
\hline 224.785 & 0 & 0 & 0 & 0 & 0 & 0 & 0.000872 & 0.000491 & 0.001077 & 0.00659 & 0.005683 & 0.011052 \\
\hline 224.685 & 0 & 0 & 0 & 0 & 0 & 0 & 0.000993 & 0.000584 & 0.001272 & 0.006871 & 0.006023 & 0.011531 \\
\hline 224.585 & 0 & 0 & 0 & 0 & 0 & 0 & 0.001133 & 0.000694 & 0.001503 & 0.007163 & 0.00638 & 0.012017 \\
\hline 224.485 & 0 & 0 & 0 & 0 & 0 & 0 & 0.001293 & 0.000824 & 0.001775 & 0.007465 & 0.006753 & 0.012511 \\
\hline 224.385 & 0 & 0 & 0 & 0 & 0 & 0 & 0.001477 & 0.000979 & 0.002095 & 0.007778 & 0.007143 & 0.01301 \\
\hline 224.285 & 0 & 0 & 0 & 0 & 0 & 0 & 0.001688 & 0.001163 & 0.002473 & 0.008102 & 0.007551 & 0.013512 \\
\hline 224.185 & 0 & 0 & 0 & 0 & 0 & 0 & 0.00193 & 0.001382 & 0.002916 & 0.008438 & 0.007976 & 0.014016 \\
\hline 224.085 & 0 & 0 & 0 & 0 & 0 & 0 & 0.002208 & 0.001641 & 0.003438 & 0.008784 & 0.008419 & 0.01452 \\
\hline 223.985 & 0 & 0 & 0 & 0 & 0 & 0 & 0.002528 & 0.001948 & 0.004048 & 0.009141 & 0.008878 & 0.015022 \\
\hline 223.885 & 0 & 0 & 0 & 0 & 0 & 0 & 0.002895 & 0.002312 & 0.004763 & 0.009509 & 0.009355 & 0.015519 \\
\hline 223.785 & 0 & 0 & 0 & 0 & 0 & 0 & 0.003316 & 0.002743 & 0.005597 & 0.009888 & 0.009847 & 0.016008 \\
\hline 223.685 & 0 & 0 & 0 & 0 & 0 & 0 & 0.003801 & 0.003252 & 0.006566 & 0.010277 & 0.010355 & 0.016488 \\
\hline 223.585 & 0 & 0 & 0 & 0 & 0 & 0 & 0.004356 & 0.003853 & 0.007688 & 0.010676 & 0.010878 & 0.016955 \\
\hline 223.485 & 0 & 0 & 0 & 0 & 0 & 0 & 0.004993 & 0.00456 & 0.008982 & 0.011085 & 0.011415 & 0.017407 \\
\hline 223.385 & 0 & 0 & 0 & 0 & 0 & 0 & 0.005721 & 0.005391 & 0.010464 & 0.011503 & 0.011964 & 0.017841 \\
\hline 223.285 & 0 & 0 & 0 & 0 & 0 & 0 & 0.006554 & 0.006363 & 0.012152 & 0.01193 & 0.012524 & 0.018254 \\
\hline 223.185 & 0 & 0 & 0 & 0 & 0 & 0 & 0.007505 & 0.007497 & 0.014058 & 0.012364 & 0.013093 & 0.018642 \\
\hline 223.085 & 0 & 0 & 0 & 0 & 0 & 0 & 0.008586 & 0.008813 & 0.016191 & 0.012805 & 0.01367 & 0.019005 \\
\hline 222.985 & 0 & 0 & 0 & 0 & 0 & 0 & 0.009812 & 0.010332 & 0.018551 & 0.013252 & 0.014252 & 0.019337 \\
\hline 222.885 & 0 & 0 & 0 & 0 & 0 & 0 & 0.011196 & 0.012075 & 0.021128 & 0.013704 & 0.014836 & 0.019638 \\
\hline
\end{tabular}




\begin{tabular}{|c|c|c|c|c|c|c|c|c|c|c|c|c|}
\hline \multirow{2}{*}{$\begin{array}{c}\text { Interval } \\
\text { (Ma) }\end{array}$} & \multicolumn{3}{|c|}{ Desmatosuchus } & \multicolumn{3}{|c|}{$\underline{\text { Smilosuchus }}$} & \multicolumn{3}{|c|}{ Typothorax } & \multicolumn{3}{|c|}{ Paratypothorax } \\
\hline & Solow & $\begin{array}{l}\text { Alroy } \\
\text { (NU) }\end{array}$ & $\begin{array}{l}\text { Alroy } \\
\text { (U) }\end{array}$ & Solow & $\begin{array}{l}\text { Alroy } \\
\text { (NU) }\end{array}$ & $\begin{array}{l}\text { Alroy } \\
\text { (U) }\end{array}$ & Solow & $\begin{array}{l}\text { Alroy } \\
\text { (NU) }\end{array}$ & $\begin{array}{l}\text { Alroy } \\
\text { (U) }\end{array}$ & Solow & $\begin{array}{l}\text { Alroy } \\
\text { (NU) }\end{array}$ & $\begin{array}{l}\text { Alroy } \\
\text { (U) }\end{array}$ \\
\hline 222.785 & 0 & 0 & 0 & 0 & 0 & 0 & 0.012752 & 0.014058 & 0.023899 & 0.01416 & 0.01542 & 0.019903 \\
\hline 222.685 & 0 & 0 & 0 & 0 & 0 & 0 & 0.01449 & 0.016292 & 0.026825 & 0.014618 & 0.016001 & 0.020132 \\
\hline 222.585 & 0 & 0 & 0 & 0 & 0 & 0 & 0.016418 & 0.018782 & 0.029848 & 0.015076 & 0.016575 & 0.020322 \\
\hline 222.485 & 0 & 0 & 0 & 0 & 0 & 0 & 0.018538 & 0.02152 & 0.03289 & 0.015533 & 0.01714 & 0.020471 \\
\hline 222.385 & 0 & 0 & 0 & 0 & 0 & 0 & 0.020845 & 0.024484 & 0.035853 & 0.015988 & 0.017691 & 0.020578 \\
\hline 222.285 & 0 & 0 & 0 & 0 & 0 & 0 & 0.023326 & 0.02763 & 0.038624 & 0.016437 & 0.018226 & 0.020642 \\
\hline 222.185 & 0 & 0 & 0 & 0 & 0 & 0 & 0.025952 & 0.030897 & 0.041079 & 0.01688 & 0.018739 & 0.020661 \\
\hline 222.085 & 0 & 0 & 0 & 0 & 0 & 0 & 0.028685 & 0.034195 & 0.043092 & 0.017314 & 0.019228 & 0.020635 \\
\hline 221.985 & 0 & 0 & 0 & 0 & 0 & 0 & 0.031468 & 0.037413 & 0.044548 & 0.017736 & 0.019688 & 0.020565 \\
\hline 221.885 & 0 & 0 & 0 & 0 & 0 & 0 & 0.034227 & 0.04042 & 0.045355 & 0.018144 & 0.020115 & 0.020451 \\
\hline 221.785 & 0 & 0 & 0 & 0 & 0 & 0 & 0.036875 & 0.043073 & 0.045452 & 0.018536 & 0.020507 & 0.020293 \\
\hline 221.685 & 0 & 0 & 0 & 0 & 0 & 0 & 0.03931 & 0.045227 & 0.044823 & 0.01891 & 0.020858 & 0.020092 \\
\hline 221.585 & 0 & 0 & 0 & 0 & 0 & 0 & 0.041424 & 0.046751 & 0.043494 & 0.019261 & 0.021166 & 0.01985 \\
\hline 221.485 & 0 & 0 & 0 & 0 & 0 & 0 & 0.043111 & 0.047541 & 0.041537 & 0.019589 & 0.021428 & 0.01957 \\
\hline 221.385 & 0 & 0 & 0 & 0 & 0 & 0 & 0.044264 & 0.04753 & 0.039056 & 0.01989 & 0.021641 & 0.019252 \\
\hline 221.285 & 0 & 0 & 0 & 0 & 0 & 0 & 0.044825 & 0.046722 & 0.036187 & 0.020162 & 0.021803 & 0.0189 \\
\hline 221.185 & 0 & 0 & 0 & 0 & 0 & 0 & 0.044738 & 0.045153 & 0.033065 & 0.020402 & 0.021912 & 0.018515 \\
\hline 221.085 & 0 & 0 & 0 & 0 & 0 & 0 & 0.043991 & 0.042915 & 0.029827 & 0.020608 & 0.021966 & 0.018102 \\
\hline 220.985 & 0 & 0 & 0 & 0 & 0 & 0 & 0.042613 & 0.04014 & 0.026594 & 0.020778 & 0.021966 & 0.017662 \\
\hline 220.885 & 0 & 0 & 0 & 0 & 0 & 0 & 0.040668 & 0.03698 & 0.023466 & 0.02091 & 0.021909 & 0.017199 \\
\hline 220.785 & 0 & 0 & 0 & 0 & 0 & 0 & 0.038251 & 0.033591 & 0.020518 & 0.021002 & 0.021798 & 0.016716 \\
\hline 220.685 & 0 & 0 & 0 & 0 & 0 & 0 & 0.035476 & 0.030123 & 0.017802 & 0.021053 & 0.021632 & 0.016217 \\
\hline 220.585 & 0 & 0 & 0 & 0 & 0 & 0 & 0.032456 & 0.026696 & 0.015344 & 0.021061 & 0.021414 & 0.015704 \\
\hline 220.485 & 0 & 0 & 0 & 0 & 0 & 0 & 0.029333 & 0.023426 & 0.01316 & 0.021026 & 0.021145 & 0.01518 \\
\hline 220.385 & 0 & 0 & 0 & 0 & 0 & 0 & 0.026206 & 0.020378 & 0.011245 & 0.020947 & 0.020827 & 0.014649 \\
\hline
\end{tabular}




\begin{tabular}{|c|c|c|c|c|c|c|c|c|c|c|c|c|}
\hline \multirow{2}{*}{$\begin{array}{c}\text { Interval } \\
\text { (Ma) }\end{array}$} & \multicolumn{3}{|c|}{ Desmatosuchus } & \multicolumn{3}{|c|}{$\underline{\text { Smilosuchus }}$} & \multicolumn{3}{|c|}{ Typothorax } & \multicolumn{3}{|c|}{ Paratypothorax } \\
\hline & Solow & $\begin{array}{l}\text { Alroy } \\
\text { (NU) }\end{array}$ & $\begin{array}{l}\text { Alroy } \\
\text { (U) }\end{array}$ & Solow & $\begin{array}{l}\text { Alroy } \\
\text { (NU) }\end{array}$ & $\begin{array}{l}\text { Alroy } \\
\text { (U) }\end{array}$ & Solow & $\begin{array}{l}\text { Alroy } \\
\text { (NU) }\end{array}$ & $\begin{array}{l}\text { Alroy } \\
\text { (U) }\end{array}$ & Solow & $\begin{array}{l}\text { Alroy } \\
\text { (NU) }\end{array}$ & $\begin{array}{l}\text { Alroy } \\
\text { (U) }\end{array}$ \\
\hline 220.285 & 0 & 0 & 0 & 0 & 0 & 0 & 0.023166 & 0.017597 & 0.009584 & 0.020824 & 0.020464 & 0.014113 \\
\hline 220.185 & 0 & 0 & 0 & 0 & 0 & 0 & 0.020286 & 0.015106 & 0.008159 & 0.020657 & 0.020059 & 0.013575 \\
\hline 220.085 & 0 & 0 & 0 & 0 & 0 & 0 & 0.017526 & 0.012846 & 0.006915 & 0.020446 & 0.019615 & 0.013038 \\
\hline 219.985 & 0 & 0 & 0 & 0 & 0 & 0 & 0.014661 & 0.010625 & 0.005734 & 0.020193 & 0.019136 & 0.012504 \\
\hline 219.885 & 0 & 0 & 0 & 0 & 0 & 0 & 0.011177 & 0.008064 & 0.004409 & 0.019899 & 0.018627 & 0.011975 \\
\hline 219.785 & 0 & 0 & 0 & 0 & 0 & 0 & 0.005065 & 0.003719 & 0.002194 & 0.019565 & 0.018091 & 0.011453 \\
\hline 219.685 & 0 & 0 & 0 & 0 & 0 & 0 & 0.001799 & 0.001421 & 0.00103 & 0.019193 & 0.017532 & 0.01094 \\
\hline 219.585 & 0 & 0 & 0 & 0 & 0 & 0 & 0.001048 & 0.000901 & 0.000771 & 0.018786 & 0.016955 & 0.010437 \\
\hline 219.485 & 0 & 0 & 0 & 0 & 0 & 0 & 0.000623 & 0.000615 & 0.000627 & 0.018346 & 0.016364 & 0.009946 \\
\hline 219.385 & 0 & 0 & 0 & 0 & 0 & 0 & 0.000468 & 0.000519 & 0.000573 & 0.017876 & 0.015763 & 0.009468 \\
\hline 219.285 & 0 & 0 & 0 & 0 & 0 & 0 & 0.000479 & 0.00054 & 0.000573 & 0.017378 & 0.015156 & 0.009004 \\
\hline 219.185 & 0 & 0 & 0 & 0 & 0 & 0 & 0.000511 & 0.000572 & 0.000572 & 0.016795 & 0.014499 & 0.008528 \\
\hline 219.085 & 0 & 0 & 0 & 0 & 0 & 0 & 0.000536 & 0.000593 & 0.000563 & 0.016192 & 0.01384 & 0.008066 \\
\hline 218.985 & 0 & 0 & 0 & 0 & 0 & 0 & 0.000554 & 0.000604 & 0.000547 & 0.015386 & 0.013036 & 0.007537 \\
\hline 218.885 & $1.88 \mathrm{E}-05$ & $1.65 \mathrm{E}-05$ & $8.98 \mathrm{E}-06$ & 0 & 0 & 0 & 0.000564 & 0.000605 & 0.000525 & 0.01394 & 0.011735 & 0.006743 \\
\hline 218.785 & 3.87E-05 & $3.36 \mathrm{E}-05$ & $1.84 \mathrm{E}-05$ & 0 & 0 & 0 & 0.000565 & 0.000595 & 0.000499 & 0.011638 & 0.009758 & 0.005585 \\
\hline 218.685 & 5.95E-05 & 5.14E-05 & 2.83E-05 & 0 & 0 & 0 & 0.000558 & 0.000577 & 0.000469 & 0.009311 & 0.007793 & 0.004447 \\
\hline 218.585 & 0.000117 & 0.0001 & 5.51E-05 & 0 & 0 & 0 & 0.000544 & 0.000552 & 0.000437 & 0.006713 & 0.005608 & 0.003196 \\
\hline 218.485 & 0.000161 & 0.000135 & $7.51 \mathrm{E}-05$ & 0 & 0 & 0 & 0.000523 & 0.000522 & 0.000403 & 0.004402 & 0.003689 & 0.002104 \\
\hline 218.385 & 0.00019 & 0.000162 & $9.08 \mathrm{E}-05$ & 0 & 0 & 0 & 0.000498 & 0.000488 & 0.000367 & 0.002374 & 0.002001 & 0.001149 \\
\hline 218.285 & 0.000203 & 0.000177 & 0.0001 & 0 & 0 & 0 & 0.00047 & 0.000451 & 0.00033 & 0.000981 & 0.000835 & 0.000491 \\
\hline 218.185 & 0.000216 & 0.000192 & 0.00011 & 0 & 0 & 0 & 0.000439 & 0.000413 & 0.000293 & 0.00039 & 0.000339 & 0.000212 \\
\hline 218.085 & 0.000245 & 0.00022 & 0.000128 & 0 & 0 & 0 & 0.000406 & 0.000374 & 0.000257 & 0.000223 & 0.000196 & 0.000131 \\
\hline 217.985 & 0.000258 & 0.000237 & 0.000141 & 0 & 0 & 0 & 0.000373 & 0.000335 & 0.000222 & 0.00016 & 0.00014 & 9.97E-05 \\
\hline 217.885 & 0.00027 & 0.000253 & 0.000154 & 0 & 0 & 0 & 0.000314 & 0.000278 & 0.00018 & 0.000157 & 0.000136 & $9.78 \mathrm{E}-05$ \\
\hline
\end{tabular}




\begin{tabular}{|c|c|c|c|c|c|c|c|c|c|c|c|c|}
\hline \multirow{2}{*}{$\begin{array}{c}\text { Interval } \\
\text { (Ma) }\end{array}$} & \multicolumn{3}{|c|}{ Desmatosuchus } & \multicolumn{3}{|c|}{$\underline{\text { Smilosuchus }}$} & \multicolumn{3}{|c|}{ Typothorax } & \multicolumn{3}{|c|}{ Paratypothorax } \\
\hline & Solow & $\begin{array}{l}\text { Alroy } \\
\text { (NU) }\end{array}$ & $\begin{array}{l}\text { Alroy } \\
\text { (U) }\end{array}$ & Solow & $\begin{array}{l}\text { Alroy } \\
\text { (NU) }\end{array}$ & $\begin{array}{l}\text { Alroy } \\
\text { (U) }\end{array}$ & Solow & $\begin{array}{l}\text { Alroy } \\
\text { (NU) }\end{array}$ & $\begin{array}{l}\text { Alroy } \\
\text { (U) }\end{array}$ & Solow & $\begin{array}{l}\text { Alroy } \\
\text { (NU) }\end{array}$ & $\begin{array}{l}\text { Alroy } \\
\text { (U) }\end{array}$ \\
\hline 217.785 & 0.000297 & 0.000283 & 0.000174 & 0 & 0 & 0 & 0.000259 & 0.000226 & 0.000143 & 0.000138 & 0.000119 & $8.83 \mathrm{E}-05$ \\
\hline 217.685 & 0.000324 & 0.000312 & 0.000196 & 0 & 0 & 0 & 0.000195 & 0.000166 & 0.000104 & $7.63 \mathrm{E}-05$ & $6.92 \mathrm{E}-05$ & $6.08 \mathrm{E}-05$ \\
\hline 217.585 & 0.000381 & 0.000366 & 0.000232 & 0 & 0 & 0 & 0.00015 & 0.000125 & $7.63 \mathrm{E}-05$ & 7.53E-05 & 6.89E-05 & $6.09 \mathrm{E}-05$ \\
\hline 217.485 & 0.000502 & 0.000469 & 0.000296 & 0 & 0 & 0 & 0.000135 & 0.000107 & $6.26 \mathrm{E}-05$ & 5.92E-05 & $5.50 \mathrm{E}-05$ & $5.31 \mathrm{E}-05$ \\
\hline 217.385 & 0.000613 & 0.00057 & 0.00036 & 0 & 0 & 0 & 0.000119 & 8.97E-05 & 5.07E-05 & 4.34E-05 & $4.26 \mathrm{E}-05$ & 4.61E-05 \\
\hline 217.285 & 0.000743 & 0.000685 & 0.000433 & 0 & 0 & 0 & $8.87 \mathrm{E}-05$ & $6.44 \mathrm{E}-05$ & $3.58 \mathrm{E}-05$ & $4.38 \mathrm{E}-05$ & $4.41 \mathrm{E}-05$ & 4.67E-05 \\
\hline 217.185 & 0.000953 & 0.000866 & 0.000543 & $3.08 \mathrm{E}-05$ & $2.38 \mathrm{E}-05$ & $1.31 \mathrm{E}-05$ & $7.58 \mathrm{E}-05$ & $5.31 \mathrm{E}-05$ & $2.87 \mathrm{E}-05$ & $4.4 \mathrm{E}-05$ & 4.57E-05 & $4.71 \mathrm{E}-05$ \\
\hline 217.085 & 0.001188 & 0.001068 & 0.000667 & $3.36 \mathrm{E}-05$ & 2.63E-05 & $1.46 \mathrm{E}-05$ & $6.34 \mathrm{E}-05$ & $4.32 \mathrm{E}-05$ & $2.29 \mathrm{E}-05$ & $2.83 \mathrm{E}-05$ & 3.39E-05 & $3.98 \mathrm{E}-05$ \\
\hline 216.985 & 0.001462 & 0.001304 & 0.000811 & $9.62 \mathrm{E}-05$ & $7.58 \mathrm{E}-05$ & $4.21 \mathrm{E}-05$ & $5.20 \mathrm{E}-05$ & $3.48 \mathrm{E}-05$ & $1.82 \mathrm{E}-05$ & $2.91 \mathrm{E}-05$ & $3.59 \mathrm{E}-05$ & 4.00E-05 \\
\hline 216.885 & 0.001818 & 0.001609 & 0.000995 & 0.000149 & 0.00012 & 6.69E-05 & $4.20 \mathrm{E}-05$ & $2.78 \mathrm{E}-05$ & $1.44 \mathrm{E}-05$ & $2.98 \mathrm{E}-05$ & $3.78 \mathrm{E}-05$ & 3.99E-05 \\
\hline 216.785 & 0.002121 & 0.001879 & 0.001163 & 0.000206 & 0.000168 & $9.40 \mathrm{E}-05$ & $1.87 \mathrm{E}-05$ & $1.23 \mathrm{E}-05$ & $6.32 \mathrm{E}-06$ & $3.05 \mathrm{E}-05$ & $3.94 \mathrm{E}-05$ & $3.95 \mathrm{E}-05$ \\
\hline 216.685 & 0.002558 & 0.002263 & 0.001396 & 0.000296 & 0.000244 & 0.000137 & 0 & 0 & 0 & $3.1 \mathrm{E}-05$ & $4.08 \mathrm{E}-05$ & $3.88 \mathrm{E}-05$ \\
\hline 216.585 & 0.00334 & 0.002919 & 0.001778 & 0.000465 & 0.000382 & 0.000215 & 0 & 0 & 0 & $3.14 \mathrm{E}-05$ & 4.18E-05 & 3.79E-05 \\
\hline 216.485 & 0.004157 & 0.003616 & 0.002188 & 0.000548 & 0.000456 & 0.000259 & 0 & 0 & 0 & 3.17E-05 & $4.25 \mathrm{E}-05$ & 3.67E-05 \\
\hline 216.385 & 0.005476 & 0.004712 & 0.00282 & 0.000606 & 0.000514 & 0.000295 & 0 & 0 & 0 & $3.19 \mathrm{E}-05$ & $4.29 \mathrm{E}-05$ & $3.53 \mathrm{E}-05$ \\
\hline 216.285 & 0.007052 & 0.006019 & 0.003573 & 0.000764 & 0.000654 & 0.000377 & 0 & 0 & 0 & 3.19E-05 & $4.28 \mathrm{E}-05$ & 3.37E-05 \\
\hline 216.185 & 0.008632 & 0.007354 & 0.004353 & 0.000958 & 0.000824 & 0.000479 & 0 & 0 & 0 & $3.18 \mathrm{E}-05$ & $4.24 \mathrm{E}-05$ & 3.19E-05 \\
\hline 216.085 & 0.010251 & 0.008742 & 0.005173 & 0.001124 & 0.000978 & 0.000574 & 0 & 0 & 0 & $3.15 E-05$ & 4.16E-05 & $3.01 \mathrm{E}-05$ \\
\hline 215.985 & 0.011638 & 0.009972 & 0.005924 & 0.001391 & 0.001215 & 0.000717 & 0 & 0 & 0 & $3.11 \mathrm{E}-05$ & 4.05E-05 & $2.82 \mathrm{E}-05$ \\
\hline 215.885 & 0.01314 & 0.011317 & 0.006753 & 0.001619 & 0.001428 & 0.000851 & 0 & 0 & 0 & $3.06 \mathrm{E}-05$ & $3.90 \mathrm{E}-05$ & 2.63E-05 \\
\hline 215.785 & 0.014521 & 0.012588 & 0.007562 & 0.001883 & 0.001674 & 0.001008 & 0 & 0 & 0 & $2.99 \mathrm{E}-05$ & 3.73E-05 & 2.44E-05 \\
\hline 215.685 & 0.015938 & 0.013914 & 0.008422 & 0.00217 & 0.001945 & 0.001184 & 0 & 0 & 0 & $2.9 \mathrm{E}-05$ & $3.54 \mathrm{E}-05$ & $2.25 \mathrm{E}-05$ \\
\hline 215.585 & 0.016868 & 0.014891 & 0.009118 & 0.002455 & 0.00222 & 0.001369 & 0 & 0 & 0 & $2.81 \mathrm{E}-05$ & $3.34 \mathrm{E}-05$ & 2.07E-05 \\
\hline 215.485 & 0.01771 & 0.015822 & 0.009815 & 0.002673 & 0.002448 & 0.001534 & 0 & 0 & 0 & 2.7E-05 & 3.12E-05 & $1.90 \mathrm{E}-05$ \\
\hline 215.385 & 0.01847 & 0.016709 & 0.010515 & 0.002917 & 0.002702 & 0.001721 & 0 & 0 & 0 & $2.59 \mathrm{E}-05$ & $2.90 \mathrm{E}-05$ & $1.73 \mathrm{E}-05$ \\
\hline
\end{tabular}




\begin{tabular}{|c|c|c|c|c|c|c|c|c|c|c|c|c|}
\hline \multirow{2}{*}{$\begin{array}{c}\text { Interval } \\
\text { (Ma) }\end{array}$} & \multicolumn{3}{|c|}{ Desmatosuchus } & \multicolumn{3}{|c|}{$\underline{\text { Smilosuchus }}$} & \multicolumn{3}{|c|}{ Typothorax } & \multicolumn{3}{|c|}{ Paratypothorax } \\
\hline & Solow & $\begin{array}{l}\text { Alroy } \\
\text { (NU) }\end{array}$ & $\begin{array}{l}\text { Alroy } \\
\text { (U) }\end{array}$ & Solow & $\begin{array}{l}\text { Alroy } \\
\text { (NU) }\end{array}$ & $\begin{array}{l}\text { Alroy } \\
\text { (U) }\end{array}$ & Solow & $\begin{array}{l}\text { Alroy } \\
\text { (NU) }\end{array}$ & $\begin{array}{l}\text { Alroy } \\
\text { (U) }\end{array}$ & Solow & $\begin{array}{l}\text { Alroy } \\
\text { (NU) }\end{array}$ & $\begin{array}{l}\text { Alroy } \\
\text { (U) }\end{array}$ \\
\hline 215.285 & 0.019219 & 0.017607 & 0.011249 & 0.003262 & 0.003037 & 0.00196 & 0 & 0 & 0 & 2.47E-05 & $2.68 \mathrm{E}-05$ & 1.57E-05 \\
\hline 215.185 & 0.019906 & 0.018473 & 0.011997 & 0.003651 & 0.003411 & 0.00223 & 0 & 0 & 0 & 2.34E-05 & 2.46E-05 & $1.43 \mathrm{E}-05$ \\
\hline 215.085 & 0.020606 & 0.019359 & 0.012787 & 0.004244 & 0.003944 & 0.002596 & 0 & 0 & 0 & 0 & 0 & 0 \\
\hline 214.985 & 0.02127 & 0.020224 & 0.013596 & 0.005832 & 0.005239 & 0.00339 & 0 & 0 & 0 & 0 & 0 & 0 \\
\hline 214.885 & 0.021848 & 0.021028 & 0.014404 & 0.008243 & 0.007175 & 0.004548 & 0 & 0 & 0 & 0 & 0 & 0 \\
\hline 214.785 & 0.022336 & 0.021761 & 0.015206 & 0.010818 & 0.009259 & 0.005803 & 0 & 0 & 0 & 0 & 0 & 0 \\
\hline 214.685 & 0.022717 & 0.022404 & 0.01599 & 0.013026 & 0.011089 & 0.006936 & 0 & 0 & 0 & 0 & 0 & 0 \\
\hline 214.585 & 0.023075 & 0.023017 & 0.016784 & 0.014488 & 0.012381 & 0.007791 & 0 & 0 & 0 & 0 & 0 & 0 \\
\hline 214.485 & 0.023312 & 0.023521 & 0.017547 & 0.015683 & 0.013501 & 0.008571 & 0 & 0 & 0 & 0 & 0 & 0 \\
\hline 214.385 & 0.023458 & 0.023932 & 0.018282 & 0.016869 & 0.014642 & 0.009382 & 0 & 0 & 0 & 0 & 0 & 0 \\
\hline 214.285 & 0.023543 & 0.02427 & 0.018996 & 0.01807 & 0.015821 & 0.010234 & 0 & 0 & 0 & 0 & 0 & 0 \\
\hline 214.185 & 0.023537 & 0.024507 & 0.019669 & 0.019171 & 0.016956 & 0.011087 & 0 & 0 & 0 & 0 & 0 & 0 \\
\hline 214.085 & 0.023473 & 0.024663 & 0.020307 & 0.020265 & 0.018114 & 0.011978 & 0 & 0 & 0 & 0 & 0 & 0 \\
\hline 213.985 & 0.023332 & 0.024723 & 0.020897 & 0.021279 & 0.019244 & 0.012883 & 0 & 0 & 0 & 0 & 0 & 0 \\
\hline 213.885 & 0.023117 & 0.024685 & 0.021431 & 0.022199 & 0.020334 & 0.013796 & 0 & 0 & 0 & 0 & 0 & 0 \\
\hline 213.785 & 0.022842 & 0.02456 & 0.021908 & 0.023046 & 0.021396 & 0.014729 & 0 & 0 & 0 & 0 & 0 & 0 \\
\hline 213.685 & 0.02251 & 0.024348 & 0.022323 & 0.023828 & 0.022432 & 0.015686 & 0 & 0 & 0 & 0 & 0 & 0 \\
\hline 213.585 & 0.022127 & 0.024055 & 0.022671 & 0.024536 & 0.02343 & 0.016661 & 0 & 0 & 0 & 0 & 0 & 0 \\
\hline 213.485 & 0.021697 & 0.023684 & 0.022949 & 0.02517 & 0.024384 & 0.017656 & 0 & 0 & 0 & 0 & 0 & 0 \\
\hline 213.385 & 0.021224 & 0.023243 & 0.023155 & 0.025722 & 0.025279 & 0.018663 & 0 & 0 & 0 & 0 & 0 & 0 \\
\hline 213.285 & 0.020715 & 0.022736 & 0.023286 & 0.026184 & 0.026101 & 0.019677 & 0 & 0 & 0 & 0 & 0 & 0 \\
\hline 213.185 & 0.020175 & 0.022171 & 0.023341 & 0.026551 & 0.026836 & 0.02069 & 0 & 0 & 0 & 0 & 0 & 0 \\
\hline 213.085 & 0.019607 & 0.021555 & 0.023321 & 0.026816 & 0.027469 & 0.021691 & 0 & 0 & 0 & 0 & 0 & 0 \\
\hline 212.985 & 0.019018 & 0.020896 & 0.023226 & 0.026978 & 0.027989 & 0.02267 & 0 & 0 & 0 & 0 & 0 & 0 \\
\hline 212.885 & 0.018412 & 0.0202 & 0.02306 & 0.027035 & 0.028383 & 0.023617 & 0 & 0 & 0 & 0 & 0 & 0 \\
\hline
\end{tabular}




\begin{tabular}{|c|c|c|c|c|c|c|c|c|c|c|c|c|}
\hline \multirow{2}{*}{$\begin{array}{c}\text { Interval } \\
\text { (Ma) }\end{array}$} & \multicolumn{3}{|c|}{ Desmatosuchus } & \multicolumn{3}{|c|}{$\underline{\text { Smilosuchus }}$} & \multicolumn{3}{|c|}{ Typothorax } & \multicolumn{3}{|c|}{ Paratypothorax } \\
\hline & Solow & $\begin{array}{l}\text { Alroy } \\
\text { (NU) }\end{array}$ & $\begin{array}{l}\text { Alroy } \\
\text { (U) }\end{array}$ & Solow & $\begin{array}{l}\text { Alroy } \\
\text { (NU) }\end{array}$ & $\begin{array}{l}\text { Alroy } \\
\text { (U) }\end{array}$ & Solow & $\begin{array}{l}\text { Alroy } \\
\text { (NU) }\end{array}$ & $\begin{array}{l}\text { Alroy } \\
\text { (U) }\end{array}$ & Solow & $\begin{array}{l}\text { Alroy } \\
\text { (NU) }\end{array}$ & $\begin{array}{l}\text { Alroy } \\
\text { (U) }\end{array}$ \\
\hline 212.785 & 0.017793 & 0.019476 & 0.022824 & 0.026986 & 0.028643 & 0.024518 & 0 & 0 & 0 & 0 & 0 & 0 \\
\hline 212.685 & 0.017166 & 0.01873 & 0.022523 & 0.026833 & 0.028762 & 0.025362 & 0 & 0 & 0 & 0 & 0 & 0 \\
\hline 212.585 & 0.016535 & 0.017969 & 0.02216 & 0.02658 & 0.028737 & 0.026134 & 0 & 0 & 0 & 0 & 0 & 0 \\
\hline 212.485 & 0.015903 & 0.0172 & 0.02174 & 0.026231 & 0.028568 & 0.026821 & 0 & 0 & 0 & 0 & 0 & 0 \\
\hline 212.385 & 0.015273 & 0.016427 & 0.02127 & 0.025793 & 0.028257 & 0.027413 & 0 & 0 & 0 & 0 & 0 & 0 \\
\hline 212.285 & 0.014648 & 0.015657 & 0.020753 & 0.025274 & 0.02781 & 0.027897 & 0 & 0 & 0 & 0 & 0 & 0 \\
\hline 212.185 & 0.014031 & 0.014893 & 0.020197 & 0.02468 & 0.027236 & 0.028264 & 0 & 0 & 0 & 0 & 0 & 0 \\
\hline 212.085 & 0.013424 & 0.01414 & 0.019606 & 0.02402 & 0.026544 & 0.028506 & 0 & 0 & 0 & 0 & 0 & 0 \\
\hline 211.985 & 0.012829 & 0.013402 & 0.018988 & 0.023305 & 0.025749 & 0.028619 & 0 & 0 & 0 & 0 & 0 & 0 \\
\hline 211.885 & 0.012247 & 0.012682 & 0.018346 & 0.022542 & 0.024864 & 0.028599 & 0 & 0 & 0 & 0 & 0 & 0 \\
\hline 211.785 & 0.011681 & 0.011981 & 0.017687 & 0.021742 & 0.023904 & 0.028446 & 0 & 0 & 0 & 0 & 0 & 0 \\
\hline 211.685 & 0.011131 & 0.011303 & 0.017017 & 0.020913 & 0.022885 & 0.028163 & 0 & 0 & 0 & 0 & 0 & 0 \\
\hline 211.585 & 0.010597 & 0.010648 & 0.016339 & 0.020063 & 0.02182 & 0.027754 & 0 & 0 & 0 & 0 & 0 & 0 \\
\hline 211.485 & 0.010081 & 0.010018 & 0.015658 & 0.019201 & 0.020725 & 0.027228 & 0 & 0 & 0 & 0 & 0 & 0 \\
\hline 211.385 & 0.009584 & 0.009413 & 0.014978 & 0.018333 & 0.019614 & 0.026594 & 0 & 0 & 0 & 0 & 0 & 0 \\
\hline 211.285 & 0.009105 & 0.008835 & 0.014304 & 0.017467 & 0.018498 & 0.025862 & 0 & 0 & 0 & 0 & 0 & 0 \\
\hline 211.185 & 0.008644 & 0.008283 & 0.013637 & 0.016608 & 0.01739 & 0.025045 & 0 & 0 & 0 & 0 & 0 & 0 \\
\hline 211.085 & 0.008202 & 0.007757 & 0.012981 & 0.015762 & 0.016298 & 0.024156 & 0 & 0 & 0 & 0 & 0 & 0 \\
\hline 210.985 & 0.007778 & 0.007258 & 0.012339 & 0.014933 & 0.015231 & 0.023207 & 0 & 0 & 0 & 0 & 0 & 0 \\
\hline 210.885 & 0.007373 & 0.006785 & 0.011713 & 0.014125 & 0.014196 & 0.022213 & 0 & 0 & 0 & 0 & 0 & 0 \\
\hline 210.785 & 0.006986 & 0.006336 & 0.011103 & 0.013341 & 0.013199 & 0.021186 & 0 & 0 & 0 & 0 & 0 & 0 \\
\hline 210.685 & 0.006617 & 0.005913 & 0.010512 & 0.012582 & 0.012244 & 0.020139 & 0 & 0 & 0 & 0 & 0 & 0 \\
\hline 210.585 & 0.006265 & 0.005514 & 0.009941 & 0.011852 & 0.011333 & 0.019082 & 0 & 0 & 0 & 0 & 0 & 0 \\
\hline 210.485 & 0.005929 & 0.005137 & 0.009391 & 0.011152 & 0.01047 & 0.018026 & 0 & 0 & 0 & 0 & 0 & 0 \\
\hline 210.385 & 0.005611 & 0.004783 & 0.008861 & 0.010482 & 0.009654 & 0.016979 & 0 & 0 & 0 & 0 & 0 & 0 \\
\hline
\end{tabular}




\begin{tabular}{|c|c|c|c|c|c|c|c|c|c|c|c|c|}
\hline \multirow{2}{*}{$\begin{array}{c}\text { Interval } \\
\text { (Ma) }\end{array}$} & \multicolumn{3}{|c|}{ Desmatosuchus } & \multicolumn{3}{|c|}{$\underline{\text { Smilosuchus }}$} & \multicolumn{3}{|c|}{ Typothorax } & \multicolumn{3}{|c|}{ Paratypothorax } \\
\hline & Solow & $\begin{array}{l}\text { Alroy } \\
\text { (NU) }\end{array}$ & $\begin{array}{l}\text { Alroy } \\
\text { (U) }\end{array}$ & Solow & $\begin{array}{l}\text { Alroy } \\
\text { (NU) }\end{array}$ & $\begin{array}{l}\text { Alroy } \\
\text { (U) }\end{array}$ & Solow & $\begin{array}{l}\text { Alroy } \\
\text { (NU) }\end{array}$ & $\begin{array}{l}\text { Alroy } \\
\text { (U) }\end{array}$ & Solow & $\begin{array}{l}\text { Alroy } \\
\text { (NU) }\end{array}$ & $\begin{array}{l}\text { Alroy } \\
\text { (U) }\end{array}$ \\
\hline 210.285 & 0.005308 & 0.004451 & 0.008353 & 0.009843 & 0.008887 & 0.015951 & 0 & 0 & 0 & 0 & 0 & 0 \\
\hline 210.185 & 0.00502 & 0.004139 & 0.007866 & 0.009235 & 0.008169 & 0.014948 & 0 & 0 & 0 & 0 & 0 & 0 \\
\hline 210.085 & 0.004747 & 0.003847 & 0.007402 & 0.008658 & 0.007497 & 0.013974 & 0 & 0 & 0 & 0 & 0 & 0 \\
\hline 209.985 & 0.004488 & 0.003573 & 0.006958 & 0.008111 & 0.006872 & 0.013036 & 0 & 0 & 0 & 0 & 0 & 0 \\
\hline
\end{tabular}


TABLE S11. AVERAGE POSTERIOR ORIGINATION PROBABILITIES GENERATED FOR REVUELTOSAURUS, CHINDESAURUS, AND MACHAEROPROSOPUS BY ALROY (2014) AND SOLOW (2016) METHODS. “NO UNDERSAMPLING” AND “UNDERSAMPLING” DENTOED RESPECTIVELY BY "NU" AND "U".

\begin{tabular}{|c|c|c|c|c|c|c|c|c|c|}
\hline \multirow{2}{*}{$\begin{array}{c}\text { Interval } \\
\text { (Ma) }\end{array}$} & \multicolumn{3}{|c|}{$\underline{\text { Revueltosaurus }}$} & \multicolumn{3}{|c|}{$\underline{\text { Chindesaurus }}$} & \multicolumn{3}{|c|}{ Machaeroprosopus } \\
\hline & Solow & $\begin{array}{l}\text { Alroy } \\
\text { (NU) }\end{array}$ & $\begin{array}{l}\text { Alroy } \\
\text { (U) }\end{array}$ & Solow & $\begin{array}{l}\text { Alroy } \\
\text { (NU) }\end{array}$ & $\begin{array}{l}\text { Alroy } \\
\text { (U) }\end{array}$ & Solow & $\begin{array}{l}\text { Alroy } \\
\text { (NU) }\end{array}$ & $\begin{array}{l}\text { Alroy } \\
\text { (U) }\end{array}$ \\
\hline & 00033 & 0.000349 & 0.000797 & 0.000191 & 0.000123 & 0.000309 & $3.65 \mathrm{E}-05$ & $1.91 \mathrm{E}-06$ & $8.2 \mathrm{E}-06$ \\
\hline & 000342 & 0.000362 & 0.000825 & 0.000199 & 0.000129 & 0.000323 & $3.82 \mathrm{E}-05$ & $2.08 \mathrm{E}-06$ & \\
\hline 224.985 & 0.000354 & 0.000376 & 0.000854 & 0.000207 & 0.000136 & 0.000337 & 3.99E-05 & 2.27E-06 & 9.57E-06 \\
\hline 224.885 & 0.000367 & 0.000391 & & & 0.000 & & 4.17E-05 & & \\
\hline 224.785 & 0.00038 & 0.000407 & 0.000915 & 0.00 & 0.000 & 0.00 & 4.37E-05 & $2.70 \mathrm{E}$ & -05 \\
\hline & 000394 & 0.00 & & & & & 57E-05 & & \\
\hline & 0.000409 & 0.00044 & 0.00 & 0.00 & 0.00 & & 4.79E-05 & & \\
\hline 224.485 & 0.000424 & 0.000458 & & 0.0002 & 0.00 & & $5.02 \mathrm{E}-05$ & $3.51 \mathrm{E}-06$ & \\
\hline 224.385 & 0.00044 & 0.000 & 0.00 & 0265 & 0.00 & & $5.26 \mathrm{E}-05$ & 3.83E-06 & $E-05$ \\
\hline & 0.000456 & 0.000 & 0.00 & 0.00 & 0.00 & 0.0 & $5.51 \mathrm{E}-05$ & & 1.67 \\
\hline & 0.000473 & 0.000516 & 0.0 & 0.00 & 0.00 & & $5.78 \mathrm{E}-05$ & & \\
\hline & 0.000491 & 0.00 & 0.00 & 0.0003 & 0.00 & & $6.06 \mathrm{E}-05$ & & \\
\hline & 0.00051 & 0.000 & & & & & $6.36 \mathrm{E}$ & & \\
\hline 223.885 & 0.00053 & 0.000584 & 0.001263 & 0.000 & 0.00 & & $6.68 \mathrm{E}-05$ & 5.98 & \\
\hline & 0.000551 & 0.000609 & 0.00131 & 0.000342 & 0.000 & 0.00 & $7.01 \mathrm{E}-05$ & $6.54 \mathrm{E}-06$ & $2.5 \mathrm{E}-05$ \\
\hline 223.685 & 0.000572 & 0.000635 & 0.00136 & 0.000357 & 0.000261 & 0.00061 & 7.37E-05 & 7.16E-06 & $2.71 \mathrm{E}-05$ \\
\hline & 0.000595 & 0.000662 & 0.001412 & 0.000373 & 0.000275 & 0.00064 & $7.75 E-05$ & 7.84E-06 & 2.94E-05 \\
\hline & 0.000618 & 0.000691 & 0.001466 & 0.00039 & 0.00029 & 0.000671 & $8.14 \mathrm{E}-05$ & & $3.2 \mathrm{E}-05$ \\
\hline & 0.000643 & 0.000721 & 0.001523 & 0.000 & 0.000 & & $8.57 \mathrm{E}-05$ & & 3.47E-05 \\
\hline 223.285 & 0.000669 & 0.000753 & & 0.000426 & 0.000323 & & $9.01 \mathrm{E}-05$ & $1.03 E-05$ & 3.77E-05 \\
\hline 223.185 & 0.000697 & 0.000786 & 0.001643 & 0.000446 & 0.000341 & 0.000776 & $9.49 \mathrm{E}-05$ & $1.13 E-05$ & 4.1E-05 \\
\hline 223.085 & 0.000725 & 0.000821 & 0.001708 & 0.000467 & 0.00036 & 0.000815 & $9.99 E-05$ & $1.24 \mathrm{E}-05$ & 4.46E-05 \\
\hline & 0.000755 & 0.000858 & & & & & 0.000105 & & 4.86E-05 \\
\hline 222.885 & 0.000787 & 0.000897 & 0.001846 & 0.000512 & 0.000402 & 0.0009 & 0.000111 & $1.49 \mathrm{E}-05$ & 5.29E-05 \\
\hline
\end{tabular}




\begin{tabular}{|c|c|c|c|c|c|c|c|c|c|}
\hline \multirow{2}{*}{$\begin{array}{c}\text { Interval } \\
\text { (Ma) }\end{array}$} & \multicolumn{3}{|c|}{ Revueltosaurus } & \multicolumn{3}{|c|}{ Chindesaurus } & \multicolumn{3}{|c|}{ Machaeroprosopus } \\
\hline & Solow & $\begin{array}{l}\text { Alroy } \\
\text { (NU) }\end{array}$ & $\begin{array}{l}\text { Alroy } \\
\text { (U) }\end{array}$ & Solow & $\begin{array}{l}\text { Alroy } \\
\text { (NU) }\end{array}$ & $\begin{array}{l}\text { Alroy } \\
\text { (U) }\end{array}$ & Solow & $\begin{array}{l}\text { Alroy } \\
\text { (NU) }\end{array}$ & $\begin{array}{l}\text { Alroy } \\
\text { (U) }\end{array}$ \\
\hline 222.785 & 0.00082 & 0.000938 & 0.00192 & 000536 & 0.000425 & 0.000946 & 0.000117 & $1.64 \mathrm{E}-05$ & $5.76 \mathrm{E}-05$ \\
\hline 222.685 & 0.000854 & 0.000981 & 0.001997 & 0.000562 & 0.000449 & 0.000994 & 0.000123 & $1.80 \mathrm{E}-05$ & $6.27 \mathrm{E}-05$ \\
\hline 222.585 & 0.000891 & 0.001026 & 0.002078 & 0.000589 & 0.000475 & 0.001046 & 0.00013 & $1.98 \mathrm{E}-05$ & $6.83 \mathrm{E}-05$ \\
\hline 222.485 & 0.000929 & 0.001073 & 0.002163 & 0.000618 & 0.0005 & 0.0011 & 0.000138 & 2.17E-05 & 7.44E-05 \\
\hline 222.385 & 0.000969 & 0.001124 & 0.002252 & 0.000648 & 0.000532 & 0.001157 & 0.000145 & $2.39 \mathrm{E}-05$ & $8.12 \mathrm{E}-05$ \\
\hline 222.285 & 0.001011 & 0.001177 & 0.002345 & 0.00068 & 0.000563 & 0.001218 & 0.000154 & 2.63E-05 & $8.85 \mathrm{E}-05$ \\
\hline 222.185 & 0.001056 & 0.001232 & 0.002442 & 0.000714 & 0.000596 & 0.001282 & 0.000162 & $2.89 \mathrm{E}-05$ & $9.66 \mathrm{E}-05$ \\
\hline 222.085 & 0.001102 & 0.001291 & 0.002544 & 0.00075 & 0.0 & 0.00135 & 0.000172 & -05 & 0.000 \\
\hline 221.985 & 0.001152 & 0.001353 & 0.002651 & 0.000788 & 0.000 & 0.001422 & 0.000182 & $3.51 \mathrm{E}-05$ & 0.000115 \\
\hline 221.885 & 0.001203 & 0.001418 & 0.002763 & 0.000828 & 0.00071 & 0.001498 & 0.000192 & 3.87E-05 & 0.000126 \\
\hline 221.785 & 0.001258 & 0.001487 & 0.00288 & 0.00087 & 0.000753 & 0.001578 & 0.000204 & $4.26 \mathrm{E}-05$ & 0.000137 \\
\hline 221.685 & 0.001315 & 0.00156 & 0.003003 & 0.000915 & 0.0007 & 0.001664 & 0.000216 & 4.70E-05 & 0.00015 \\
\hline 221.585 & 0.001375 & 0.001 & 0.003133 & 0.000963 & & 0.001754 & 0.000229 & & 0.000164 \\
\hline 221.485 & 0.001439 & 0.001718 & 0.003268 & 0.001014 & 0.00 & 0.00185 & 0.000243 & -05 & 0.000 \\
\hline 221.385 & 0.001506 & 0.001804 & 0.00341 & 0.001067 & 0.0009 & 0.001951 & 0.000258 & -05 & 0.000197 \\
\hline 221.285 & 0.001577 & 0.001894 & 0.003559 & 0.001124 & 0.001015 & 0.002059 & 0.000274 & $6.98 \mathrm{E}-05$ & 0.000216 \\
\hline 221.185 & 0.001651 & 0.00199 & 0.003715 & 0.001184 & 0.001079 & 0.002173 & 0.000291 & 7.72E-05 & 0.000236 \\
\hline 221.085 & 0.00173 & 0.002091 & 0.003879 & 0.001249 & 0.001146 & 0.002294 & 0.00031 & 8.53E-05 & 0.000259 \\
\hline 220.985 & 0.001813 & 0.00 & 0.004051 & 0.001317 & 0.001 & 0.002422 & 0.00033 & 9.44 & 0.000284 \\
\hline 220.885 & 0.001901 & 0.002311 & 0.004232 & 0.001389 & 0.001296 & 0.002558 & 0.000351 & 0.000104 & 0.000311 \\
\hline 220.785 & 0.001994 & 0.002431 & 0.004421 & 0.001466 & 0.001379 & 0.002702 & 0.000374 & 0.000116 & 0.000341 \\
\hline 220.685 & 0.002091 & 0.002557 & 0.00462 & 0.001547 & 0.001468 & 0.002856 & 0.000398 & 0.000128 & 0.000374 \\
\hline & 0.002195 & & 0.004828 & 0.001634 & & 0.003018 & 0.000425 & & 0.000411 \\
\hline 220.485 & 0.002304 & 0.002833 & 0.005047 & 0.001726 & 0.001664 & 0.003191 & 0.000454 & 0.000157 & 0.000451 \\
\hline 220.385 & 0.002419 & 0.002983 & 0.005276 & 0.001825 & 0.001772 & 0.003374 & 0.000484 & 0.000174 & 0.000496 \\
\hline
\end{tabular}




\begin{tabular}{|c|c|c|c|c|c|c|c|c|c|}
\hline \multirow{2}{*}{$\begin{array}{c}\text { Interval } \\
\text { (Ma) }\end{array}$} & \multicolumn{3}{|c|}{ Revueltosaurus } & \multicolumn{3}{|c|}{ Chindesaurus } & \multicolumn{3}{|c|}{ Machaeroprosopus } \\
\hline & Solow & $\begin{array}{l}\text { Alroy } \\
\text { (NU) }\end{array}$ & $\begin{array}{l}\text { Alroy } \\
\text { (U) }\end{array}$ & Solow & $\begin{array}{l}\text { Alroy } \\
\text { (NU) }\end{array}$ & $\begin{array}{l}\text { Alroy } \\
\text { (U) }\end{array}$ & Solow & $\begin{array}{l}\text { Alroy } \\
\text { (NU) }\end{array}$ & $\begin{array}{l}\text { Alroy } \\
\text { (U) }\end{array}$ \\
\hline 20.285 & 0.002542 & 0.003142 & 0.005516 & 0.001929 & 0.001888 & 0.003569 & 0.000518 & 0.000194 & 0.000545 \\
\hline 220.185 & 0.002671 & 0.00331 & 0.005767 & 0.00204 & 0.002013 & 0.003776 & 0.000553 & 0.000215 & 0.000599 \\
\hline 220.085 & 0.002807 & 0.003488 & 0.00603 & 0.002159 & 0.002146 & 0.003996 & 0.000592 & 0.000239 & 0.000659 \\
\hline 219.985 & 0.002952 & 0.003677 & 0.006306 & 0.002285 & 0.0022 & 0.00423 & 0.000634 & 0.000265 & 0.000725 \\
\hline 219.885 & 0.003105 & 0.003877 & 0.006595 & 0.00242 & 0.002442 & 0.004478 & 0.000679 & 295 & 0.000798 \\
\hline 219.7 & 0.003267 & 0.004089 & 0.006896 & 0.002563 & 0.002606 & 0.004742 & 0.000728 & 328 & 0.000879 \\
\hline 219.685 & 0.003439 & 0.004313 & 0.007211 & 0.002716 & 0.002782 & 0.005023 & 0.000781 & 0.000365 & 0.000967 \\
\hline 219.585 & 0.003621 & 0.00 & 0.00754 & $0.002 \varepsilon$ & 0.0 & 0.005 & 0.000838 & & 0.001066 \\
\hline 219.485 & 0.003814 & 0.004803 & 0.007884 & 0.003053 & 0.003 & 0.005637 & 0.000901 & 52 & 0.001174 \\
\hline 219.385 & 0.004019 & 0.00507 & 0.008241 & 0.003239 & 0.00 & 0.005973 & 0.000968 & 03 & 0.001294 \\
\hline 219.285 & 0.004235 & 0.005352 & 0.008613 & 0.003437 & 0.003624 & 0.006329 & 0.001042 & 0.000 & 0.001427 \\
\hline 219.185 & 0.004465 & 0.005651 & 0.009 & 0.003649 & 0.003 & 0.006707 & 0.001122 & 0.00 & 0.001573 \\
\hline 219.085 & 0.004709 & 0.005968 & 0.009402 & 0.003 & & 0.007 & 0.001209 & & 0.001735 \\
\hline 218.985 & 0.004968 & 0.006303 & 0.009818 & 0.004117 & 0.00 & 0.007 & 0.001303 & & 0.001914 \\
\hline 218.885 & 0.005242 & 0.006657 & 0.010248 & 0.004375 & 0.004 & 0.007 & 0.001406 & 868 & 0.002112 \\
\hline 218.785 & 0.005533 & 0.007031 & 0.010692 & 0.004651 & 0.005077 & 0.008458 & 0.001519 & 0.000 & 0.00233 \\
\hline 218.685 & 0.005841 & 0.007426 & 0.01115 & 0.004946 & 0.005435 & 0.00896 & 0.001641 & 0.001083 & 0.002571 \\
\hline 218.585 & 0.006169 & 0.007843 & 0.01162 & 0.005261 & 0.00582 & 0.00949 & 0.001775 & 0.00121 & 0.002837 \\
\hline 218.485 & 0.006516 & & 0.012101 & 0.005598 & 0.00 & 0.010048 & 0.001922 & 0.00 & 0.00313 \\
\hline 218.385 & 0.006885 & 0.008745 & 0.012593 & 0.005958 & 0.0066 & 0.010634 & 0.002082 & 0.001 & 0.003453 \\
\hline 218.285 & 0.007275 & 0.009232 & 0.013094 & 0.006344 & 0.007151 & 0.011249 & 0.002257 & 0.00169 & 0.003809 \\
\hline 218.185 & 0.00769 & 0.009744 & 0.013601 & 0.006756 & 0.007661 & 0.011892 & 0.002449 & 0.00189 & 0.004201 \\
\hline 218.085 & 0.008129 & & 0.014114 & 0.007196 & & 0.012564 & 0.002659 & & 0.004632 \\
\hline 217.985 & 0.008594 & 0.010841 & 0.014629 & 0.007667 & 0.00879 & 0.013262 & 0.00289 & 0.002365 & 0.005106 \\
\hline 217.885 & 0.009087 & 0.011427 & 0.015144 & 0.00817 & 0.009414 & 0.013986 & 0.003142 & 0.002645 & 0.005625 \\
\hline
\end{tabular}




\begin{tabular}{|c|c|c|c|c|c|c|c|c|c|}
\hline \multirow{2}{*}{$\begin{array}{c}\text { Interval } \\
\text { (Ma) }\end{array}$} & \multicolumn{3}{|c|}{ Revueltosaurus } & \multicolumn{3}{|c|}{ Chindesaurus } & \multicolumn{3}{|c|}{ Machaeroprosopus } \\
\hline & Solow & $\begin{array}{l}\text { Alroy } \\
\text { (NU) }\end{array}$ & $\begin{array}{l}\text { Alroy } \\
\text { (U) }\end{array}$ & Solow & $\begin{array}{l}\text { Alroy } \\
\text { (NU) }\end{array}$ & $\begin{array}{l}\text { Alroy } \\
(U)\end{array}$ & Solow & $\begin{array}{l}\text { Alroy } \\
\text { (NU) }\end{array}$ & $\begin{array}{l}\text { Alroy } \\
\text { (U) }\end{array}$ \\
\hline 217.785 & 0.009608 & 0.012038 & 0.015656 & 0.008707 & 0.01008 & 0.014734 & 0.00342 & 0.00296 & 0.006194 \\
\hline 217.685 & 0.010159 & & 0.016161 & 0.009281 & 0.010789 & 0.015502 & 0.003725 & & 0.006815 \\
\hline 17.585 & & & 0.016656 & 0.009892 & & 0.016287 & 0.004059 & & .007494 \\
\hline & 0.011355 & & 0.017137 & 0.010544 & & 0.017086 & 0.004427 & & 008232 \\
\hline 217.385 & 0.012002 & 0.014711 & 0.0176 & 0.011238 & 3187 & 0.017892 & 0.004832 & & 009033 \\
\hline 217.285 & 12683 & 429 & 0.01804 & 1976 & & 01 & & & \\
\hline 217.185 & & & 0.018449 & & & & & & \\
\hline 217.085 & & & & & & 0.020287 & 0.006303 & & \\
\hline 216. & 0.014888 & 0.01 & 0.019164 & 0.014425 & & 0.021042 & 0.006894 & & 012918 \\
\hline 216.885 & 0.015691 & 0.018369 & 0.0194 & 0.015336 & 0.0 & 0.021 & 0.007 & & 63 \\
\hline 216.785 & 0.016541 & 0.01 & 0.0197 & & & & & & \\
\hline 216.685 & 0.017421 & & 0.019936 & & & 0.023 & 0.00 & & \\
\hline 216.585 & & & 0.02009 & & 0.02 & 0.023 & 96 & & \\
\hline 216.485 & 0.019243 & 0.022303 & 0.021228 & 483 & 0.02 & 0.024 & 0.01 & & 0.019273 \\
\hline 216.385 & 0.020129 & 0.02193 & 0.020222 & 0.02056 & 0.02355 & 0.024535 & 0.011859 & & 0.020695 \\
\hline 216.285 & & & & & & & & & \\
\hline & & & & & & & & & \\
\hline & 0.022899 & 0.023608 & 0.019949 & 0.02399 & 0.026623 & 0.0250 & 0.015507 & 115 & 0.025042 \\
\hline & 0.023837 & 0.024074 & 0.019747 & 0.02517 & 0.027542 & 0.025031 & 0.016927 & 0.019837 & 0.026452 \\
\hline & 0.024752 & 0.02447 & 0.019483 & & 0.028364 & 0.024863 & 0.018453 & 0.021 & 0.027803 \\
\hline 215.785 & & & & & & & & & \\
\hline & 0.026376 & & 0.018752 & 0.028433 & & 0.024122 & 0.021813 & & 0.030226 \\
\hline 215.585 & 0.027072 & 0.025057 & 0.018296 & 0.029351 & 0.029956 & 0.023563 & 0.023635 & 0.02744 & 0.031244 \\
\hline 215.485 & 0.027728 & 0.025083 & 0.017805 & 0.030205 & 0.03019 & 0.022907 & 0.025535 & 0.029409 & 0.032098 \\
\hline 215.385 & 0.028271 & 0.025 & 0.017264 & 0.030915 & 0.030232 & 0.022143 & 0.027495 & 0.031344 & 0.032767 \\
\hline
\end{tabular}




\begin{tabular}{|c|c|c|c|c|c|c|c|c|c|}
\hline \multirow{2}{*}{$\begin{array}{c}\text { Interval } \\
\text { (Ma) }\end{array}$} & \multicolumn{3}{|c|}{ Revueltosaurus } & \multicolumn{3}{|c|}{ Chindesaurus } & \multicolumn{3}{|c|}{ Machaeroprosopus } \\
\hline & Solow & $\begin{array}{l}\text { Alroy } \\
\text { (NU) }\end{array}$ & $\begin{array}{l}\text { Alroy } \\
\text { (U) }\end{array}$ & Solow & $\begin{array}{l}\text { Alroy } \\
\text { (NU) }\end{array}$ & $\begin{array}{l}\text { Alroy } \\
\text { (U) }\end{array}$ & Solow & $\begin{array}{l}\text { Alroy } \\
\text { (NU) }\end{array}$ & $\begin{array}{l}\text { Alroy } \\
\text { (U) }\end{array}$ \\
\hline 15.285 & 0.028727 & 0.024826 & 0.01669 & .031497 & 0.030102 & 0.021296 & 0.02949 & 0.033205 & 0.033231 \\
\hline 215.185 & 0.029002 & 0.024521 & 0.016066 & 0.031852 & 0.029752 & 0.020356 & 0.031488 & 0.03495 & 0.033477 \\
\hline 215.085 & 0.029183 & 0.024144 & 0.015428 & 0.03206 & 0.029245 & 0.019371 & 0.03345 & 0.036536 & 0.033498 \\
\hline 214.985 & 0.029199 & 0.023652 & 0.01476 & 0.032045 & 0.028 & 0.018333 & 0.035331 & 0.037921 & 0.033294 \\
\hline 214.885 & 0.029023 & 0.024069 & 0.015104 & 0.031777 & 0.027656 & 0.017254 & 0.037078 & 063 & 0.032869 \\
\hline 214.785 & 0.028608 & 0.022332 & 0.013348 & 0.03121 & 0.026551 & 0.016127 & 0.038636 & 929 & 0.032232 \\
\hline 214.685 & 0.028081 & 0.021561 & 0.012638 & 0.030472 & 0.025365 & 0.015027 & 0.039947 & 189 & 0.031398 \\
\hline 214.585 & 0.027401 & $0.02 C$ & 0.011939 & 0.029529 & 0.0 & 0.013 & 0.040954 & & 0.030377 \\
\hline 214.485 & 0.02653 & 0.019834 & 0.01123 & 0.02835 & 0.022 & 0.012875 & 0.041605 & 09 & 0.029179 \\
\hline 214.385 & 0.025252 & 0.018703 & 0.010437 & 0.026729 & 0.02 & 0.011741 & 0.041836 & 123 & 0.027799 \\
\hline 214.285 & 0.023093 & 0.017 & 0.009374 & 0.024215 & $0.018 \varepsilon$ & 0.01037 & 0.041641 & 0.039258 & 0.026237 \\
\hline 214.185 & 0.01872 & 0.013678 & 0.007481 & 0.019504 & 0.015 & 0.008202 & 0.041031 & 0.038 & 0.024506 \\
\hline 214.085 & 0.01301 & 0.01 & 0.006237 & 0.013522 & & 0.005 & 0.039988 & & 0.022606 \\
\hline 213.985 & 0.007615 & 0.005601 & 0.003039 & 0.007951 & 0.00 & 0.003377 & 0.038486 & 11 & 0.020554 \\
\hline 213.885 & 0.003315 & 0.002454 & 0.001331 & 0.003543 & 0.002758 & 0.001571 & 0.036519 & 808 & 0.018418 \\
\hline 213.785 & 0.00095 & 0.00065 & 0.000353 & 0.001144 & 0.0009 & 0.000594 & 0.033471 & 0.028 & 0.016006 \\
\hline 213.685 & 0.000292 & 0.000166 & $9.08 \mathrm{E}-05$ & 0.000484 & 0.000426 & 0.000351 & 0.02765 & 0.023135 & 0.012756 \\
\hline 213.585 & 0.000122 & & 1.97E-05 & 0.000324 & 0.0003 & 0.000305 & 0.017551 & 0.014575 & 0.007958 \\
\hline 213.485 & & & $3.54 \mathrm{E}-07$ & 0.000313 & 0.00 & & 0.007119 & 0.006044 & 0.003311 \\
\hline 213.385 & 0.000108 & $2.2 \mathrm{E}-07$ & 5.49E-07 & 0.000331 & 0.000348 & 0.00032 & 0.002151 & 0.00 & 0.000998 \\
\hline 213.285 & 0.000117 & $3.51 \mathrm{E}-07$ & 8.53E-07 & 0.00035 & 0.000362 & 0.000322 & 0.000732 & 0.000584 & 0.000333 \\
\hline 213.185 & 0.000126 & $5.58 \mathrm{E}-07$ & $1.32 \mathrm{E}-06$ & 0.000368 & 0.000362 & 0.000317 & 0.000569 & 0.000447 & 0.000252 \\
\hline & 0.000137 & & $2.05 E-06$ & & & & 0.000505 & & 0.000217 \\
\hline 212.985 & 0.000149 & $1.41 \mathrm{E}-06$ & 3.17E-06 & 0.000373 & 0.000267 & 0.000258 & 0.000274 & 0.000223 & 0.000123 \\
\hline 212.885 & 0.000162 & $2.24 \mathrm{E}-06$ & $4.90 \mathrm{E}-06$ & 0.000393 & 0.00029 & 0.000279 & 0.000121 & 0.000101 & $5.6 \mathrm{E}-05$ \\
\hline
\end{tabular}




\begin{tabular}{|c|c|c|c|c|c|c|c|c|c|}
\hline \multirow{2}{*}{$\begin{array}{c}\text { Interval } \\
\text { (Ma) }\end{array}$} & \multicolumn{3}{|c|}{$\underline{\text { Revueltosaurus }}$} & \multicolumn{3}{|c|}{ Chindesaurus } & \multicolumn{3}{|c|}{ Machaeroprosopus } \\
\hline & Solow & $\begin{array}{l}\text { Alroy } \\
\text { (NU) }\end{array}$ & $\begin{array}{l}\text { Alroy } \\
\text { (U) }\end{array}$ & Solow & $\begin{array}{l}\text { Alroy } \\
\text { (NU) }\end{array}$ & $\begin{array}{l}\text { Alroy } \\
\text { (U) }\end{array}$ & Solow & $\begin{array}{l}\text { Alroy } \\
\text { (NU) }\end{array}$ & $\begin{array}{l}\text { Alroy } \\
\text { (U) }\end{array}$ \\
\hline 212.785 & 0.000176 & $3.55 \mathrm{E}-06$ & $7.53 \mathrm{E}-06$ & 0.000378 & 0.00029 & 0.000285 & 7.82E-05 & $6.80 \mathrm{E}-05$ & $3.74 \mathrm{E}-05$ \\
\hline 212.685 & 0.000191 & 0.001019 & 0.001046 & 0.000364 & 0.000292 & 0.000289 & 0 & 0 & 0 \\
\hline 212.585 & 0.000169 & $8.82 \mathrm{E}-06$ & $1.74 \mathrm{E}-05$ & 0.000388 & 0.000322 & 0.000305 & 0 & 0 & 0 \\
\hline 212.485 & 0.000185 & $1.38 \mathrm{E}-05$ & 2.61E-05 & 0.000331 & 0.000296 & 0.000287 & 0 & 0 & 0 \\
\hline 212.385 & 0.000202 & $2.13 E-05$ & $3.81 \mathrm{E}-05$ & 0.000357 & 0.000323 & 0.00029 & 0 & 0 & 0 \\
\hline 212.285 & 0.00022 & $3.23 E-05$ & $5.42 \mathrm{E}-05$ & 0.000379 & 0.00034 & 0.000283 & 0 & 0 & 0 \\
\hline 212.185 & 0.00024 & $4.78 \mathrm{E}-05$ & $7.38 \mathrm{E}-05$ & 0.000396 & 0.000346 & 0.000267 & 0 & 0 & 0 \\
\hline 212.085 & 0.000261 & $6.82 \mathrm{E}-05$ & $9.50 \mathrm{E}-05$ & 0.000406 & 0.001343 & 0.001255 & 0 & 0 & 0 \\
\hline 211.985 & 0.000282 & $9.22 \mathrm{E}-05$ & 0.000114 & 0.000358 & 0.000328 & 0.000233 & 0 & 0 & 0 \\
\hline 211.885 & 0.000305 & 0.000116 & 0.000125 & 0.000299 & 0.000279 & 0.000224 & 0 & 0 & 0 \\
\hline 211.785 & 0.000218 & 0.001147 & 0.001159 & 0.000234 & 0.000298 & 0.000286 & 0 & 0 & 0 \\
\hline 211.685 & 0.000184 & 0.000139 & 0.000114 & 0.000163 & 0.000337 & 0.000333 & 0 & 0 & 0 \\
\hline 211.585 & 0.000216 & 0.000131 & $9.62 \mathrm{E}-05$ & 8.65E-05 & 0.000313 & 0.000262 & 0 & 0 & 0 \\
\hline 211.485 & 0.000256 & 0.000113 & 7.93E-05 & $7.95 \mathrm{E}-05$ & 0.000196 & 0.000138 & 0 & 0 & 0 \\
\hline 211.385 & 0.000307 & $9.66 \mathrm{E}-05$ & $7.28 \mathrm{E}-05$ & 0 & 0 & 0 & 0 & 0 & 0 \\
\hline 211.285 & 0.000302 & $3.5 \mathrm{E}-05$ & $5.84 \mathrm{E}-05$ & 0 & 0 & 0 & 0 & 0 & 0 \\
\hline 211.185 & 0.00041 & 0.000106 & 0.000166 & 0 & 0 & 0 & 0 & 0 & 0 \\
\hline 211.085 & 0.000543 & 0.000284 & 0.000388 & 0 & 0 & 0 & 0 & 0 & 0 \\
\hline 210.985 & 0.000686 & 0.000556 & 0.000608 & 0 & 0 & 0 & 0 & 0 & 0 \\
\hline 210.885 & 0.000802 & 0.000633 & 0.000538 & 0 & 0 & 0 & 0 & 0 & 0 \\
\hline 210.785 & 0.000848 & 0.00141 & 0.001316 & 0 & 0 & 0 & 0 & 0 & 0 \\
\hline 210.685 & 0.000398 & 0.001014 & 0.001034 & 0 & 0 & 0 & 0 & 0 & 0 \\
\hline 210.585 & 0.00026 & 0.001014 & 0.001034 & 0 & 0 & 0 & 0 & 0 & 0 \\
\hline 210.485 & 0.000111 & 0 & 0 & 0 & 0 & 0 & 0 & 0 & 0 \\
\hline 210.385 & 0.000271 & 0 & 0 & 0 & 0 & 0 & 0 & 0 & 0 \\
\hline
\end{tabular}




\begin{tabular}{|c|c|c|c|c|c|c|c|c|c|}
\hline \multirow{2}{*}{$\begin{array}{c}\text { Interval } \\
\text { (Ma) }\end{array}$} & \multicolumn{3}{|c|}{$\underline{\text { Revueltosaurus }}$} & \multicolumn{3}{|c|}{$\underline{\text { Chindesaurus }}$} & \multicolumn{3}{|c|}{ Machaeroprosopus } \\
\hline & Solow & $\begin{array}{l}\text { Alroy } \\
\text { (NU) }\end{array}$ & $\begin{array}{l}\text { Alroy } \\
\text { (U) }\end{array}$ & Solow & $\begin{array}{l}\text { Alroy } \\
\text { (NU) }\end{array}$ & $\begin{array}{l}\text { Alroy } \\
\text { (U) }\end{array}$ & Solow & $\begin{array}{l}\text { Alroy } \\
\text { (NU) }\end{array}$ & $\begin{array}{l}\text { Alroy } \\
\text { (U) }\end{array}$ \\
\hline 210.285 & 0.000529 & 0.001014 & 0.001034 & 0 & 0 & 0 & 0 & 0 & 0 \\
\hline 210.185 & 0 & 0 & 0 & 0 & 0 & 0 & 0 & 0 & 0 \\
\hline 210.085 & 0 & 0 & 0 & 0 & 0 & 0 & 0 & 0 & 0 \\
\hline 209.985 & 0 & 0 & 0 & 0 & 0 & 0 & 0 & 0 & 0 \\
\hline
\end{tabular}




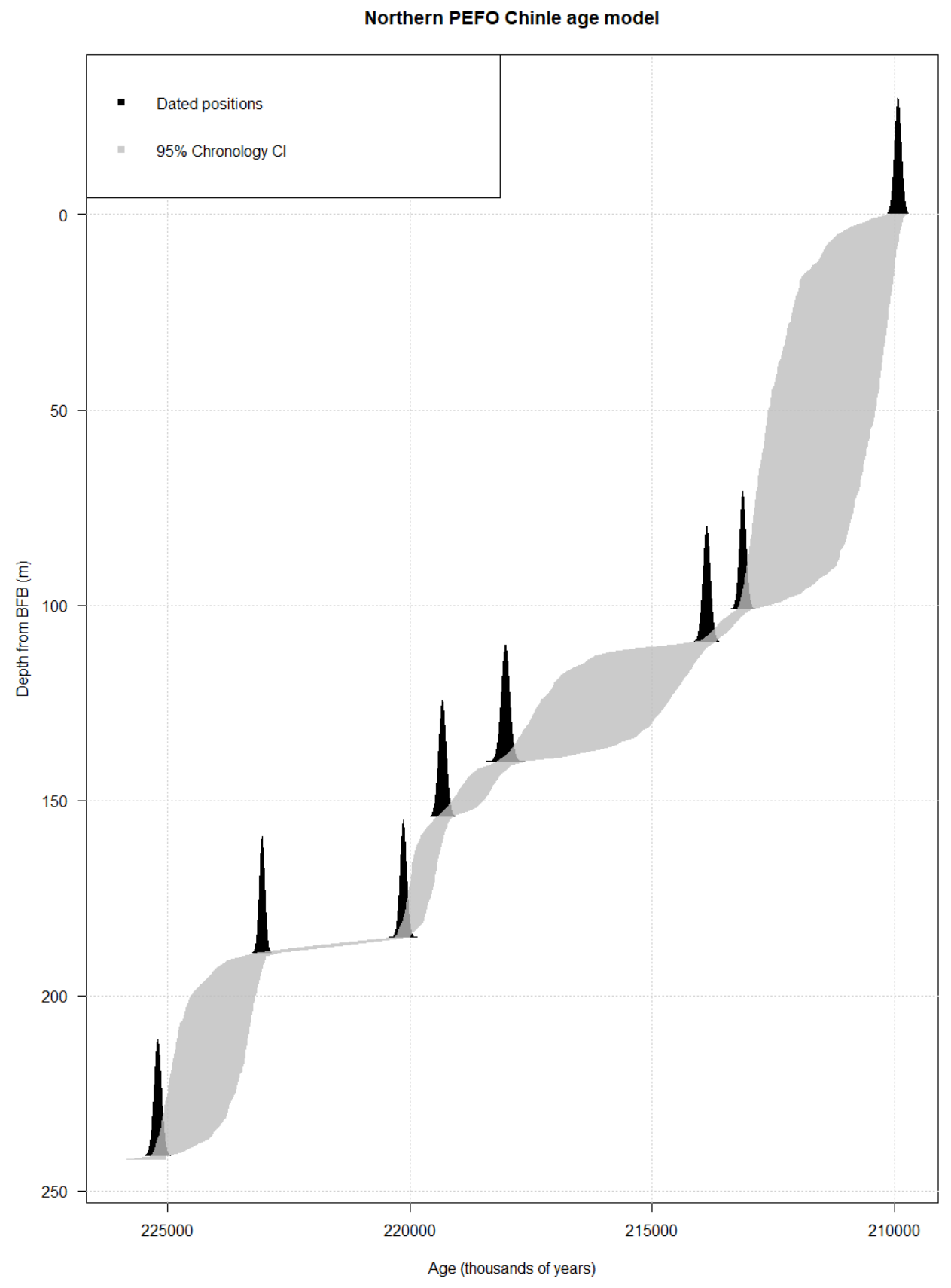

Figure S1. A Bayesian age-depth model of northern PEFO. Normal distributions (black) represent U-Pb dates, with width proportional to analytical uncertainty, while the grey field between them represents a 95\% credible interval on the sedimentation history of the Chinle in PEFO. Temporal control over the system is proportional to the width of that field at a given stratigraphic level. Depth is given relative to the Black Forest Bed (upper Petrified Forest Member), the source of the youngest $\mathrm{U}-\mathrm{Pb}$ date in the model. A separate age model (Figure S2) is used for southern PEFO, differing only in the substitution of the date P57-C ( $213.63 \pm 0.130 \mathrm{Ma})$ for KWI $(213.87 \pm 0.078 \mathrm{Ma})$. See Table S1 for model inputs, and Table S3 for diagnostics. 


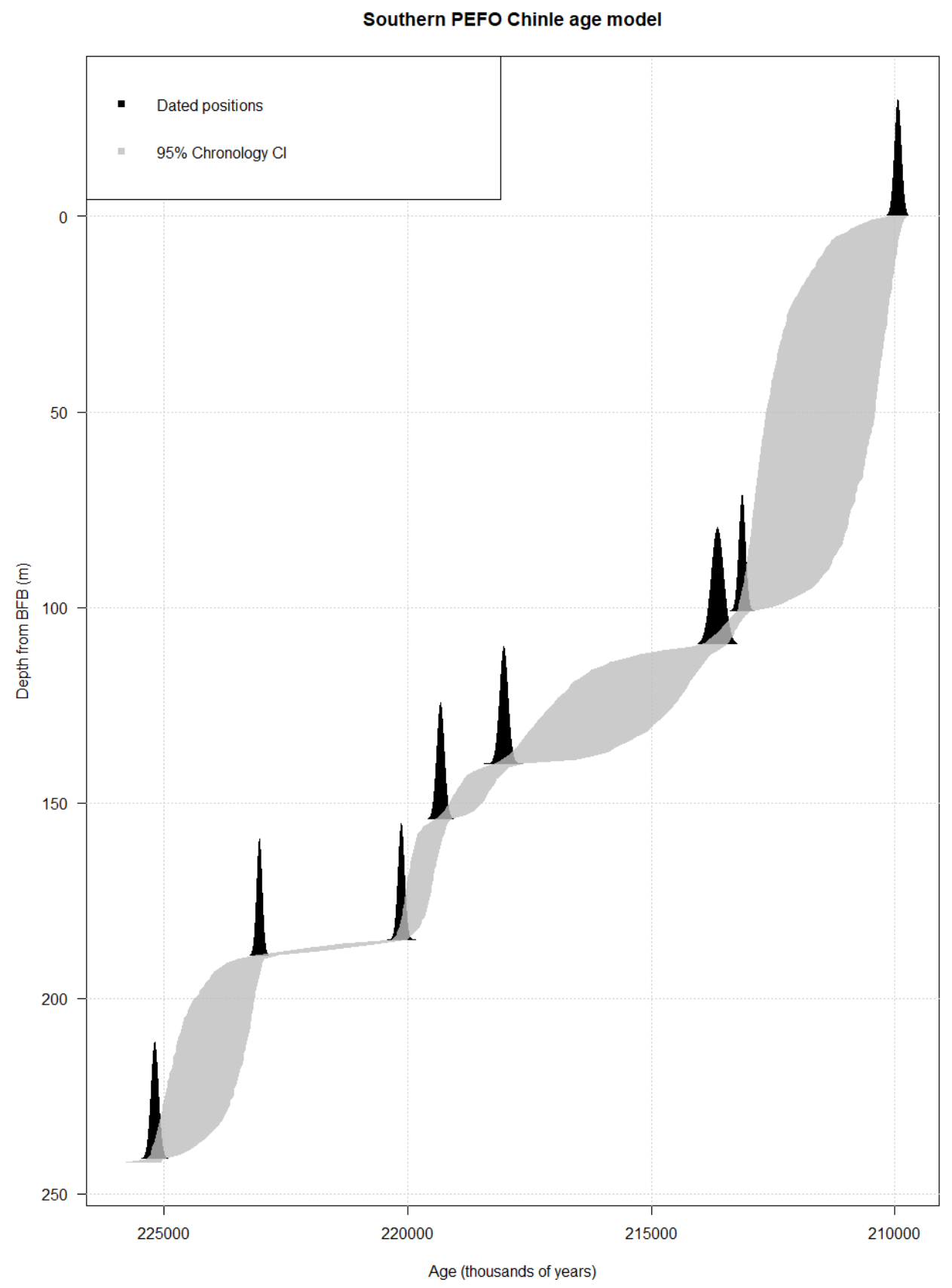

Figure S2. A Bayesian age-depth model of southern PEFO. Normal distributions (black) represent U-Pb dates, with width proportional to analytical uncertainty, while the grey field between them represents a 95\% credible interval on the sedimentation history of the Chinle in PEFO. Temporal control over the system is proportional to the width of that field at a given stratigraphic level. Depth is given relative to the Black Forest Bed (upper Petrified Forest Member), the source of the youngest U-Pb date in the model. A separate age model (Figure S1) is used for northern PEFO, differing only in the substitution of the date KWI $(213.87 \pm 0.078 \mathrm{Ma})$ for P57-C (213.63 $\pm 0.130 \mathrm{Ma})$. See Table S2 for model inputs, and Table S4 for diagnostics. 


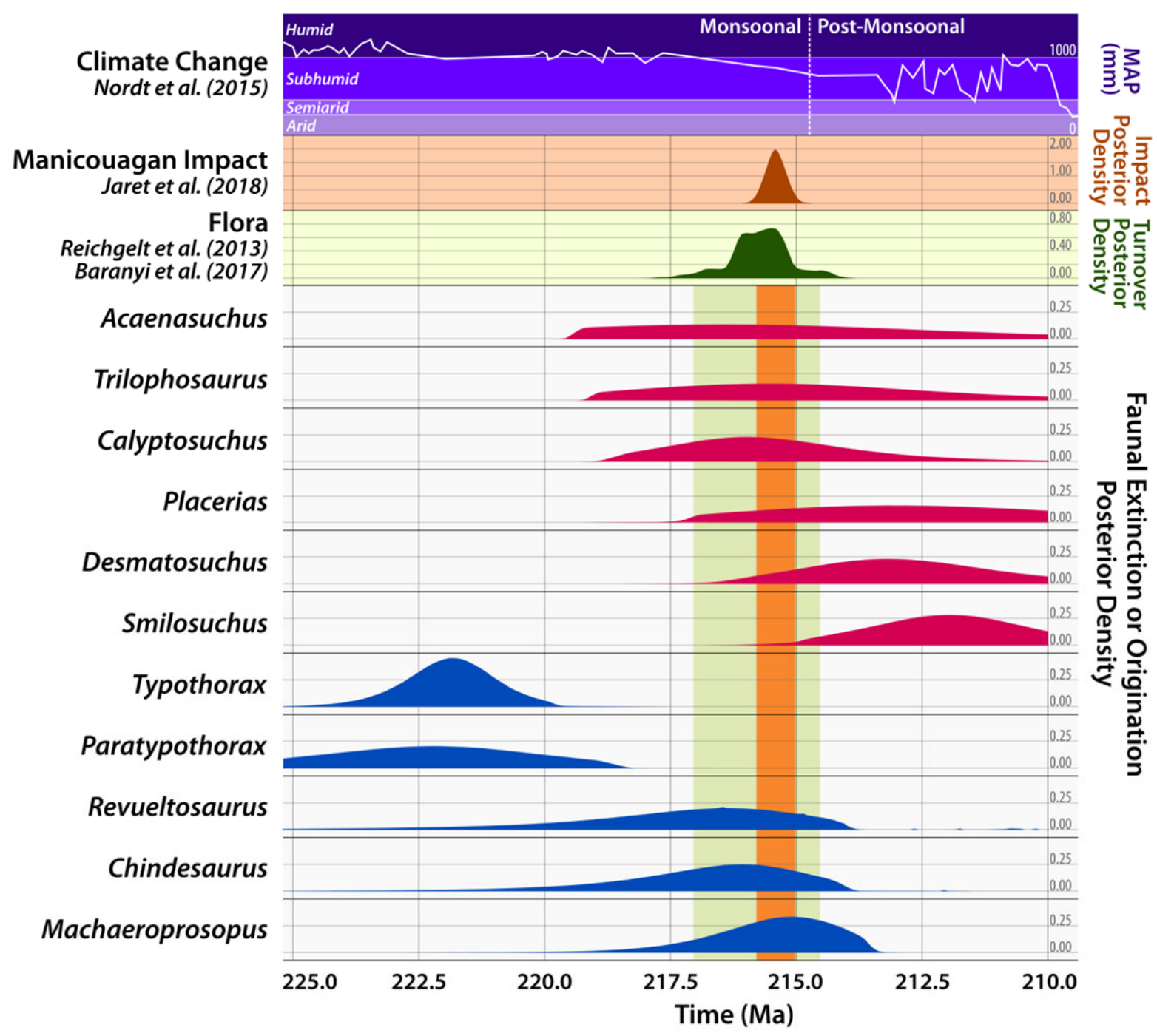

Figure S3. Posterior probability density functions of extinction and origination produced by the Alroy (2014) algorithm assuming undersampling applied in 1000 simulations to 11 PEFO tetrapod genera. Pink and blue densities respectively refer to extinction and origination. Temporal overlap between taxon densities indicates a non-zero probability that extinctions and/or originations were synchronous; Table S8 and Figure S4 list these probabilities. Chinle mean annual precipitation (MAP) record of Nordt et al. (2015) shown above. Also above are posterior probability densities of floral turnover (green; Reichgelt et al., 2013; Baranyi et al., 2017) and Manicouagan impact (orange); vertically-oriented green and orange fields (below) delineate respective $95 \%$ highest posterior density regions. 


\begin{tabular}{|c|c|c|c|c|c|c|c|c|c|c|c|c|c|}
\hline & 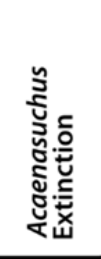 & 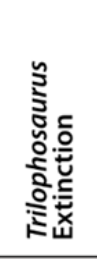 & 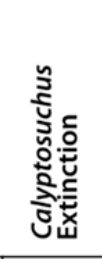 & 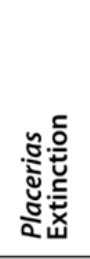 & 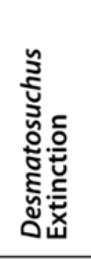 & 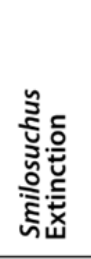 & 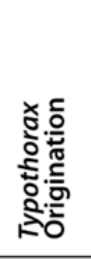 & 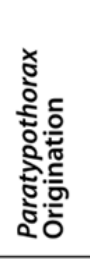 & 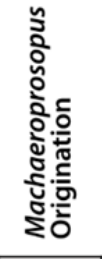 & 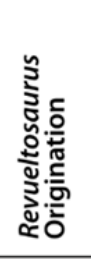 & 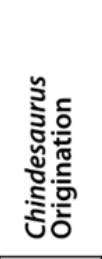 & $\begin{array}{r}\text { 힘 } \\
\text { 끈 } \\
\text { 는 }\end{array}$ & 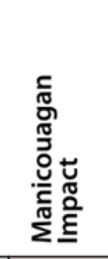 \\
\hline $\begin{array}{r}\text { Acaenasuchus } \\
\text { Extinction }\end{array}$ & & $1.15 \%$ & $1.23 \%$ & $0.98 \%$ & $0.96 \%$ & $0.81 \%$ & $0.01 \%$ & $0.07 \%$ & $1.27 \%$ & $1.06 \%$ & $1.16 \%$ & $1.34 \%$ & $6.67 \%$ \\
\hline $\begin{array}{r}\text { Trilophosaurus } \\
\text { Extinction }\end{array}$ & $1.15 \%$ & & $1.34 \%$ & $1.09 \%$ & $1.08 \%$ & $0.87 \%$ & $0.00 \%$ & $0.03 \%$ & $1.43 \%$ & $1.07 \%$ & $1.21 \%$ & $1.54 \%$ & $7.88 \%$ \\
\hline $\begin{array}{r}\text { Calyptosuchus } \\
\text { Extinction }\end{array}$ & $1.23 \%$ & $1.34 \%$ & & $1.06 \%$ & $0.95 \%$ & $0.59 \%$ & $0.00 \%$ & $0.01 \%$ & $1.81 \%$ & $1.34 \%$ & $1.55 \%$ & $2.16 \%$ & $11.08 \%$ \\
\hline $\begin{array}{r}\text { Placerias } \\
\text { Extinction }\end{array}$ & $0.98 \%$ & $1.09 \%$ & $1.06 \%$ & & $1.50 \%$ & $1.51 \%$ & $0.00 \%$ & $0.00 \%$ & $1.14 \%$ & $0.62 \%$ & $0.74 \%$ & $1.15 \%$ & $6.29 \%$ \\
\hline $\begin{array}{r}\text { Desmatosuchus } \\
\text { Extinction }\end{array}$ & $0.96 \%$ & $1.08 \%$ & $0.95 \%$ & $1.50 \%$ & & $1.74 \%$ & $0.00 \%$ & $0.00 \%$ & $1.02 \%$ & $0.43 \%$ & $0.52 \%$ & $0.79 \%$ & $4.91 \%$ \\
\hline $\begin{array}{r}\text { Smilosuchus } \\
\text { Extinction }\end{array}$ & $0.81 \%$ & $0.87 \%$ & $0.59 \%$ & $1.51 \%$ & $1.74 \%$ & & $0.00 \%$ & $0.00 \%$ & $0.39 \%$ & $0.14 \%$ & $0.16 \%$ & $0.16 \%$ & $0.78 \%$ \\
\hline $\begin{array}{l}\text { Typothorax } \\
\text { Origination }\end{array}$ & $0.01 \%$ & $0.00 \%$ & $0.00 \%$ & $0.00 \%$ & $0.00 \%$ & $0.00 \%$ & & $1.88 \%$ & $0.02 \%$ & $0.30 \%$ & $0.17 \%$ & $0.00 \%$ & $0.00 \%$ \\
\hline $\begin{array}{c}\text { Paratypothorax } \\
\text { Origination }\end{array}$ & $0.07 \%$ & $0.03 \%$ & $0.01 \%$ & $0.00 \%$ & $0.00 \%$ & $0.00 \%$ & $1.88 \%$ & & $0.03 \%$ & $0.34 \%$ & $0.21 \%$ & $0.00 \%$ & $0.00 \%$ \\
\hline $\begin{array}{r}\text { Machaeroprosopus } \\
\text { Origination }\end{array}$ & $1.27 \%$ & $1.43 \%$ & $1.81 \%$ & $1.14 \%$ & $1.02 \%$ & $0.39 \%$ & $0.02 \%$ & $0.03 \%$ & & $1.46 \%$ & $1.73 \%$ & $2.74 \%$ & $15.96 \%$ \\
\hline $\begin{array}{r}\text { Revueltosaurus } \\
\text { Origination }\end{array}$ & $1.06 \%$ & $1.07 \%$ & $1.34 \%$ & $0.62 \%$ & $0.43 \%$ & $0.14 \%$ & $0.30 \%$ & $0.34 \%$ & $1.46 \%$ & & $1.51 \%$ & $1.81 \%$ & $8.88 \%$ \\
\hline $\begin{array}{c}\text { Chindesaurus } \\
\text { Origination }\end{array}$ & $1.16 \%$ & $1.21 \%$ & $1.55 \%$ & $0.74 \%$ & $0.52 \%$ & $0.16 \%$ & $0.17 \%$ & $0.21 \%$ & $1.73 \%$ & $1.51 \%$ & & $2.25 \%$ & $11.40 \%$ \\
\hline $\begin{array}{r}\text { Floral } \\
\text { Turnover }\end{array}$ & $1.34 \%$ & $1.54 \%$ & $2.16 \%$ & $1.15 \%$ & $0.79 \%$ & $0.16 \%$ & $0.00 \%$ & $0.00 \%$ & $2.74 \%$ & $1.81 \%$ & $2.25 \%$ & & $34.08 \%$ \\
\hline $\begin{array}{r}\text { Manicouagan } \\
\text { Impact }\end{array}$ & $6.67 \%$ & $7.88 \%$ & $11.08 \%$ & $6.29 \%$ & $4.91 \%$ & $0.78 \%$ & $0.00 \%$ & $0.00 \%$ & $15.96 \%$ & $8.88 \%$ & $11.40 \%$ & $34.08 \%$ & \\
\hline
\end{tabular}

Figure S4. Probability of synchroneity of paired Chinle biotic events calculated with Alroy (2014) method assuming undersampling of taxa. Probability of synchroneity with Manicouagan impact is posterior probability at $215.40 \pm 0.20 \mathrm{Ma}$; all others are summation of joint probabilities across full time series. 